\title{
Curved fronts of bistable reaction-diffusion equations with nonlinear convection
}

\author{
Hui-Ling Niu ${ }^{1 *}$ (D) and Jiayin Liu'
}

${ }^{\text {"Correspondence: }}$ niuhling08@163.com

${ }^{1}$ School of Mathematics and Information Science, North Minzu University, Yinchuan, Ningxia 750021, People's Republic of China

\begin{abstract}
This paper is concerned with traveling curved fronts of bistable reaction-diffusion equations with nonlinear convection in a two-dimensional space. By constructing super- and subsolutions, we establish the existence of traveling curved fronts. Furthermore, we show that the traveling curved front is globally asymptotically stable.

MSC: 35K55; 35C07; 35B35; 35B40

Keywords: Traveling curved front; Reaction-diffusion equation; Nonlinear convection; Bistable nonlinearity; Stability
\end{abstract}

\section{Introduction}

In this paper, we consider traveling wave solutions of the following reaction-diffusion equations with a nonlinear convection term:

$$
u_{t}+(g(u))_{y}=\Delta u+f(u), \quad(x, y) \in \mathbb{R}^{2}, t>0,
$$

where $f$ is the nonlinear reaction term and $(g(u))_{y}$ is the nonlinear convection term. In general, the term $(g(u))_{y}$ represents a convective or advective phenomenon, with $g^{\prime}(u)$ denoting a nonlinear velocity function. As a matter of fact, reaction-diffusion equations with convection term are widely used to model some reaction-diffusion processes taking place in moving media such as fluids, for example, combustion, atmospheric chemistry, and plankton distributions in the sea, see Berestycki [1], Cencini et al. [6], Gilding and Kersner [21], Murray [41], and the references therein. Of particular interest is the influence of advection terms on the propagation of traveling wave fronts, which were studied by many researchers, see Berestycki [1], Crooks [8-10], Crooks and Mascia [11], Crooks and Toland [12], Crooks and Tsai [13], Gilding [20], Gilding and Kersner [21], Malaguti and Marcelli [36, 37], Malaguti et al. [38], Volpert et al. [52].

In this paper we assume that $f \in C^{2}(\mathbb{R})$ satisfies the following conditions:

(F) (i) $f(0)=f(1)=0, f^{\prime}(0)<0, f^{\prime}(1)<0$;

(ii) $\{r \in[0,1]: f(r)=0\}=\{0, \lambda, 1\}$ with $f^{\prime}(\lambda)>0$;

(iii) $\int_{0}^{1} f(r) \mathrm{d} r>0$;

(iv) $f(r)<0, f^{\prime}(r)<0$ for $r>1 ; f(r)>0, f^{\prime}(r)<0$ for $r<0$.

(c) The Author(s) 2020. This article is licensed under a Creative Commons Attribution 4.0 International License, which permits use, sharing, adaptation, distribution and reproduction in any medium or format, as long as you give appropriate credit to the original author(s) and the source, provide a link to the Creative Commons licence, and indicate if changes were made. The images or other third party material in this article are included in the article's Creative Commons licence, unless indicated otherwise in a credit line to the material. If material is not included in the article's Creative Commons licence and your intended use is not permitted by statutory regulation or exceeds the permitted use, you will need to obtain permission directly from the copyright holder. To view a copy of this licence, visit http://creativecommons.org/licenses/by/4.0/. 
A typical example of $\operatorname{such} f$ is the cubic function, namely

$$
f(u)=u(u-a)(1-u)
$$

where $a \in\left(0, \frac{1}{2}\right)$ is a given number. In addition, we assume that the flux $g$ satisfies the following condition:

(G) $g(r) \in C^{2+\gamma_{0}}(\mathbb{R}), \gamma_{0} \in(0,1) ; g^{\prime \prime}(r) \leq 0$ for $r \in[0,1]$.

It is obvious that the functions $g(u)=\rho u(1-u)$ and $g(u)=-\rho u^{2}$ satisfy assumption (G), where $\rho>0$ is a positive constant.

For each $\theta \in[0,2 \pi]$, a planar traveling front of (1.1) with direction $\theta$ means a function $u(x, y, t)=U_{\theta}(X), X=x \cos \theta+y \sin \theta+c_{\theta} t$ satisfying

$$
\left\{\begin{array}{l}
-U_{\theta}^{\prime \prime}+\left(c_{\theta}+g^{\prime}\left(U_{\theta}\right) \sin \theta\right) U_{\theta}^{\prime}-f\left(U_{\theta}\right)=0, \quad X \in \mathbb{R} \\
U_{\theta}(-\infty)=0, \quad U_{\theta}(+\infty)=1
\end{array}\right.
$$

where $c_{\theta} \in \mathbb{R}$ is called the wave speed. It is obvious that the existence of the solution pair $\left(U_{\theta}, c_{\theta}\right)$ satisfying (1.2) is equivalent to the existence of traveling wave fronts of the following equation in a one-dimensional space:

$$
v_{t}+\sin \theta(g(v))_{x}=v_{x x}+f(v), \quad x \in \mathbb{R}, t>0,
$$

which has been extensively studied. In 1998, Crooks and Toland [12] considered traveling wave fronts of the more general reaction-diffusion-convection system

$$
u_{t}=D u_{x x}+G\left(u, u_{x}\right) u_{x}+F(u), \quad u(x, t) \in \mathbb{R}^{N}, x \in \mathbb{R}, t \in[0, \infty)
$$

where $D$ is a positive-definite diagonal matrix, $F: \mathbb{R}^{N} \rightarrow \mathbb{R}^{N}$ is continuously differentiable and is of bistable type, $G$ is a continuously differentiable, diagonal-matrix-valued function on $\mathbb{R}^{N} \times \mathbb{R}^{N}$, and there exist continuous functions $\beta, \gamma:[0, \infty) \rightarrow[0, \infty)$ such that, for each $u, v \in \mathbb{R}^{N}, G$ satisfies

$$
\|G(u, v)\| \leq \gamma(\|u\|)(1+\beta(\|v\|)),
$$

where $\beta$ is increasing and $\beta(p) / p \rightarrow 0$ as $p \rightarrow \infty$. They showed that there exists a unique speed $c$ for which (1.3) has an increasing traveling front $\phi$ satisfying

$$
D \phi^{\prime \prime}+c \phi^{\prime}+G\left(\phi, \phi^{\prime}\right) \phi^{\prime}+F(\phi)=0
$$

and connecting two stable equilibria of (1.3). Furthermore, Crooks [8] showed the global stability of traveling front $\phi$ if the initial-value $u_{0}(x)$ is bounded, uniformly continuously differentiable and such that $\left\|\phi(x)-u_{0}(x)\right\|$ is small when $|x|$ is large.

Later, Crooks [9] studied the existence and stability of traveling-front solutions for the following gradient-dependent system:

$$
u_{t}=D u_{x x}+f\left(u, u_{x}\right), \quad x \in \mathbb{R}, t>0, u(x, t) \in \mathbb{R}^{N},
$$


where $D$ is a positive-definite diagonal matrix and $f$ is a "monostable" function. Crooks [9] showed that if $f$ satisfies some given conditions, then there exists a critical wave speed $c^{*} \in \mathbb{R}$ such that there exists a monotone traveling front solution if and only if $c \geq c^{*}$. Furthermore, the stability of traveling front solutions for system (1.4) was proved.

It should be emphasized that a special interest is to consider the case that the diffusion coefficient $D$ of (1.3) and (1.4) is vanished. In 1997, Mascia [39] established the existence of entropy traveling fronts for the balance law

$$
u_{t}+(g(u))_{x}=f(u), \quad x \in \mathbb{R}, t>0, u(x, t) \in \mathbb{R},
$$

where $g$ is a convex function while $f$ is bistable or monostable. In 2000, Mascia [40] proved the existence of entropy traveling front solutions for (1.5) with nonconvex flux $g$ and monostable reaction $f$, that is, the flux $g$ is assumed to be smooth and is allowed to have finitely many points of inflection.

Thanks to Crooks [9] and Mascia [39, 40], Crooks and Mascia [11] considered the convergence as $\varepsilon \rightarrow 0$ of traveling front speeds for the parabolic equation

$$
u_{t}+(g(u))_{x}=\varepsilon u_{x x}+f(u)
$$

to front speeds for the balance law (1.5). They assumed that the flux $g$ is smooth and may have points of inflection and the reaction term $f$ is of monostable type, with simple zeroes at 0 and 1 and negative in between. They proved that the minimal speed $c^{*}$ of fronts for (1.5) defined by using entropy criteria coincides with the vanishing-diffusion limit of the minimal speeds $c_{\varepsilon}^{*}$ for (1.6). Afterwards, Crooks [10] established the $L^{1}(\mathbb{R})$-convergence of corresponding traveling-front profiles $w_{\varepsilon}$ with speed $c_{\varepsilon}$ (minimal or non-minimal speed) and $w_{\varepsilon}(0)=1 / 2$ for (1.6) in the limit $\varepsilon \rightarrow 0$. Namely,

$$
w_{\varepsilon} \rightarrow w \quad \text { in } L^{1}(\mathbb{R})
$$

as $\varepsilon \rightarrow 0$, where $w$ is the profile of the unique entropy traveling-front solution of (1.5) with speed $c$ (minimal or non-minimal speed) and $w(0)=1 / 2$. More recently, Crooks and Tsai [13] established the existence and uniqueness of entire solutions for both monostable and bistable nonlinearity. Especially, they also considered the case that $\varepsilon \rightarrow 0$.

Assume that assumptions (F) and (G) hold. It follows from [12] that, for each fixed direction $\theta \in(0, \pi / 2)$, there exist a unique wave speed $c=c_{\theta}$ and a unique function $U_{\theta}(\cdot)$ (up to translation) satisfying (1.2). Furthermore, $U_{\theta}^{\prime}(X)>0$ for $X \in \mathbb{R}$. In contrast to that, for the reaction-diffusion equation without advection, the planar wave speed $c_{\theta}$ of (1.1) depends on the direction $\theta \in(0, \pi / 2)$. Instead of planar traveling wave fronts, in this paper we consider non-planar traveling wave fronts of (1.1) in a two-dimensional space. To do it, in the following we set $\theta \in\left(0, \frac{\pi}{2}\right)$ satisfying the following assumption:

(C) $c_{\theta}+g^{\prime}(r) \sin \theta>0$ for any $r \in[0,1]$.

Here we would like to point out that assumption $(\mathrm{C})$ is reasonable. We only consider the function $g(u)=\rho u(1-u)$ with $\rho>0$. In fact, it follows from assumption (F) that $c_{0}>0$, where $c_{0}$ is independent of the function $g(u)$. Then the function $v(x, t)=U_{\theta}\left(x+\left(c_{\theta}+\right.\right.$ $\rho \sin \theta) t$ ) is a supersolution of the following equation:

$$
v_{t}=v_{x x}+f(v), \quad x \in \mathbb{R}, t>0 .
$$


Since $U_{0}\left(x+c_{0} t-\xi-\sigma \delta\left(1-e^{-\beta t}\right)\right)-\delta e^{-\beta t}$ with suitable constants $\sigma>0, \delta>0$, and $\beta>0$ is a subsolution of the last equation (see $[8,48,57]$ ), then for sufficiently large $\xi>0$ the comparison principle yields

$$
U_{\theta}\left(x+\left(c_{\theta}+\rho \sin \theta\right) t\right) \geq U_{0}\left(x+c_{0} t+\xi-\sigma \delta\left(1-e^{-\beta t}\right)\right)-\delta e^{-\beta t}, \quad \forall x \in \mathbb{R}, t>0 .
$$

Using this inequality, we can get $c_{\theta}+\rho \sin \theta \geq c_{0}>0$. It is clear that

$$
c_{\theta}+g^{\prime}(u) \sin \theta=c_{\theta}+\rho \sin \theta-2 u \rho \sin \theta \geq c_{0}-2 u \rho \sin \theta>0
$$

for any $u \in[0,1]$ if either $\rho>0$ or $\theta \in\left(0, \frac{\pi}{2}\right)$ is small enough. Thus, we have either that assumption (C) holds for any $\theta \in\left(0, \frac{\pi}{2}\right)$ if $\rho>0$ is small enough, or for the fixed $\rho>0$, assumption $(\mathrm{C})$ holds for $\theta \in\left(0, \frac{\pi}{2}\right)$ small enough.

Assume that $(\mathrm{F})$ and $(\mathrm{G})$ hold. Let $\theta \in\left(0, \frac{\pi}{2}\right)$ satisfy $(\mathrm{C})$. Let $\left(U_{\theta}(\cdot), c_{\theta}\right)$ be defined by (1.2). Let $s_{\theta}=\frac{c_{\theta}}{\sin \theta}$. Then we have

$$
\left\{\begin{array}{l}
-U_{\theta}^{\prime \prime}+\left(c_{\theta}+\frac{c_{\theta}}{s_{\theta}} g^{\prime}\left(U_{\theta}\right)\right) U_{\theta}^{\prime}-f\left(U_{\theta}\right)=0, \quad U_{\theta}^{\prime}(X)>0, X \in \mathbb{R} \\
U_{\theta}(-\infty)=0, \quad U_{\theta}(+\infty)=1 .
\end{array}\right.
$$

From Crooks and Toland [12, Theorem 3.6], we know that there exist positive constants $C_{1}$ and $\beta_{1}$ such that

$$
\left|U_{\theta}(X)-1\right|+\left|U_{\theta}(-X)\right|+\left|U_{\theta}^{\prime}( \pm X)\right|+\left|U_{\theta}^{\prime \prime}( \pm X)\right| \leq C_{1} e^{-\beta_{1} X}, \quad \forall X \geq 0
$$

Set $u(x, y, t)=w(x, z, t)$ with $z=y+s_{\theta} t$, then equation (1.1) reduces to

$$
w_{t}-w_{x x}-w_{z z}+\left(s_{\theta}+g^{\prime}(w)\right) w_{z}-f(w)=0, \quad(x, z) \in \mathbb{R}^{2}, t>0 .
$$

To establish the existence of non-planar traveling wave fronts of (1.1) in a two-dimensional space, we need to find a function $v(x, z)$ satisfying

$$
\mathcal{L}[v]:=-v_{x x}-v_{z z}+\left(s_{\theta}+g^{\prime}(v)\right) v_{z}-f(v)=0, \quad(x, z) \in \mathbb{R}^{2} .
$$

Moreover, to give the stability of the non-planar traveling wave front $v\left(x, y+s_{\theta} t\right)$ of (1.1), we need to consider the initial problem of equation (1.9). As said by Crooks [9, p. 59], $B U C^{1}\left(\mathbb{R}^{2}\right)$ is a suitable space for the initial data $u_{0}(x, y)$ due to the nonlinear convection. Namely, we consider the stability of the non-planar traveling wave front $v\left(x, y+s_{\theta} t\right)$ of (1.1) with initial value $u_{0} \in B U C^{1}\left(\mathbb{R}^{2}\right)$. Let

$$
m_{*}:=\cot \theta .
$$

It is obvious that $m_{*}=\sqrt{s_{\theta}^{2}-c_{\theta}^{2}} / c_{\theta}$ when $c_{\theta}>0$. Then $U_{\theta}\left(\frac{1}{\sin \theta}(z+x \cot \theta)\right)$ and $U_{\theta}\left(\frac{1}{\sin \theta}(z-\right.$ $x \cot \theta)$ ) are two planar traveling wave fronts of (1.1). Let

$$
\begin{aligned}
v^{-}(x, z) & :=\max \left\{U_{\theta}\left(\frac{1}{\sin \theta}(z+x \cot \theta)\right), U_{\theta}\left(\frac{1}{\sin \theta}(z-x \cot \theta)\right)\right\} \\
& =U_{\theta}\left(\frac{1}{\sin \theta}(z+|x| \cot \theta)\right),
\end{aligned}
$$


where $(x, z) \in \mathbb{R}^{2}$. It is clear that $v_{z}^{-}(x, z)>0$ for all $(x, z) \in \mathbb{R}^{2}$. We now describe the main results of this paper.

Theorem 1.1 Assume that $(\mathrm{F})$ and $(\mathrm{G})$ hold. Let $\theta \in\left(0, \frac{\pi}{2}\right)$ satisfy assumption (C). Let $s_{\theta}=\frac{c_{\theta}}{\sin \theta}$. Then there exists a solution $u(x, y, t)=v_{*}\left(x, y+s_{\theta} t\right)$ of (1.1) satisfying (1.10) and

$$
\begin{aligned}
& \lim _{R \rightarrow \infty} \sup _{x^{2}+z^{2} \geq R^{2}}\left|v_{*}(x, z)-v^{-}(x, z)\right|=0, \\
& 0 \leq v^{-}(x, z)<v_{*}(x, z) \leq 1,
\end{aligned}
$$

where $z=y+s_{\theta} t, v^{-}(x, z)$ is defined in (1.11). Furthermore, for any initial value $u_{0} \in$ $B U C^{1}\left(\mathbb{R}^{2}\right)$ satisfying

$$
u_{0}(x, z) \geq v^{-}(x, z)
$$

and

$$
\lim _{R \rightarrow \infty} \sup _{x^{2}+z^{2} \geq R^{2}}\left|u_{0}(x, z)-v^{-}(x, z)\right|=0
$$

the solution $u\left(x, y, t ; u_{0}\right)$ of $(1.1)$ with initial value $u_{0}$ satisfies

$$
\lim _{t \rightarrow \infty}\left\|u\left(x, y, t ; u_{0}\right)-v_{*}\left(x, y+s_{\theta} t\right)\right\|_{L^{\infty}\left(\mathbb{R}^{2}\right)}=0 .
$$

In the following, we call $v\left(x, y+s_{\theta} t\right)$ defined in Theorem 1.1 a traveling curved front of (1.1). The shapes and the contour lines of the traveling curved front $v$ are similar to Figs. 1 and 2 of Wang [54, p. 2432] (see also Ninomiya and Taniguchi [43]). From Theorem 1.1, we find that traveling curved front $v$ satisfying (1.10) and (1.12) is unique. In the following, we only give the proof of Theorem 1.1 for the case $c_{\theta}>0$. In fact, for the case $c_{\theta} \leq 0$, Theorem 1.1 can be proved by that for the case $c_{\theta}>0$. Now we suppose that Theorem 1.1 has been proved for the case $c_{\theta}>0$. Fix $\theta \in(0, \pi / 2)$ satisfying $(C)$. Suppose that $c_{\theta} \leq 0$. Denote $\tilde{c}_{\theta}:=\frac{1}{2}\left(c_{\theta}+\min _{r \in[0,1]} g^{\prime}(r) \sin \theta\right)>0$. Define $\tilde{g}(u)=\frac{c_{\theta}-\tilde{c}_{\theta}}{\sin \theta} u+g(u)$. Consider a new equation:

$$
\tilde{u}_{t}+(\tilde{g}(\tilde{u}))_{y}=\Delta \tilde{u}+f(\tilde{u}), \quad(x, y) \in \mathbb{R}^{2}, t>0 .
$$

Clearly, for the solution $u(x, y, t)$ of (1.1), the function $\tilde{u}(x, y, t):=u\left(x, y-\frac{c_{\theta}-\tilde{c}_{\theta}}{\sin \theta} t, t\right)$ is a solution of (1.15). In particular, the function $\tilde{U}_{\theta}\left(x \cos \theta+y \sin \theta+\tilde{c}_{\theta} t\right):=U_{\theta}(x \cos \theta+y \sin \theta+$ $\left.\tilde{c}_{\theta} t\right)$ is also a traveling wave front of (1.15) along the direction $\theta \in(0, \pi / 2)$. Because of $\tilde{c}_{\theta}+\tilde{g}^{\prime}(r) \sin \theta=c_{\theta}+g^{\prime}(r) \sin \theta>0$ for all $r \in[0,1]$, we can get a traveling curved front $\tilde{v}_{*}\left(x, y+\tilde{s}_{\theta} t\right)$ for equation (1.15) by Theorem 1.1 for the case $c_{\theta}>0$. Let $v_{*}\left(x, y+s_{\theta} t\right):=$ $\tilde{v}_{*}\left(x, y+s_{\theta} t\right)$, then $v_{*}$ is a traveling curved front of (1.1) with speed $s_{\theta}$ which satisfies all the conditions in Theorem 1.1. Thus we complete the proof of Theorem 1.1 for the case $c_{\theta} \leq 0$.

Here we would like to point out that the results of Theorem 1.1 have been obtained by Ninomiya and Taniguchi $[43,44]$ when the nonlinear advection is absent. Similar results 
were also established for bistable reaction-diffusion systems and time-periodic reactiondiffusion equations, see [54, 60]. In fact, recently many researchers have paid attention to non-planar traveling wave solutions for the following reaction-diffusion equations:

$$
u_{t}(\mathbf{x}, t)=\Delta u(\mathbf{x}, t)+f(u(\mathbf{x}, t)), \quad \mathbf{x} \in \mathbb{R}^{N}, t>0,
$$

with various reaction terms $f$, where $N \geq 2$. We refer to $[2,24,25]$ for conical traveling wave fronts of (1.16) with ignition nonlinearity, $[7,26,27]$ for conical traveling wave fronts of (1.16) with bistable nonlinearity, [33, 47, 49-51] for pyramidal traveling wave fronts of (1.16) with bistable nonlinearity, and [3,28,32] for multi-dimensional traveling wave fronts of (1.16) with Fisher-KPP nonlinearity. For more results on non-planar traveling wave solutions of reaction-diffusion equations, we refer to $[4,5,15-17,22,23,29-31,42$, $53,56]$; for reaction-diffusion systems, we refer to $[45,46,58,59]$.

Here we would like to mention that the main method of this paper comes from Ninomiya and Taniguchi [43] and Wang [54]. Nevertheless, to the best of our knowledge, this paper is the first to consider traveling curved fronts for a reaction-diffusion equation with nonlinear convection in $\mathbb{R}^{2}$. This paper is organized as follows: In Sect. 2, we prove the existence of the traveling curved front $v$ by constructing an appropriate supersolution of (1.10). In Sect. 3, we show the asymptotic stability of the traveling curved front $v$, namely, we prove (1.14).

In the remainder of this paper we always assume that $(\mathrm{F})$ and $(\mathrm{G})$ hold and $\theta \in\left(0, \frac{\pi}{2}\right)$ satisfies assumption (C). Moreover, we also assume that $c_{\theta}>0$. Let $\left(U_{\theta}(\cdot), c_{\theta}\right)$ be defined by (1.2), and let $s_{\theta}:=\frac{c_{\theta}}{\sin \theta}>0$. In this case, we also have

$$
\nu^{-}(x, z):=U_{\theta}\left(\frac{c_{\theta}}{s_{\theta}}\left(z+m_{*}|x|\right)\right)
$$

For the sake of convenience, in the sequel we always denote $\left(U_{\theta}(\cdot), c_{\theta}\right)$ and $s_{\theta}$ by $(U(\cdot), c)$ and $s$, respectively.

\section{Existence}

In this section we show the existence of traveling curved fronts of (1.1).

It follows from Ninomiya and Taniguchi [43] that there exists a unique function $\varphi(x)$ with asymptotic lines $y=m_{*}|x|$ satisfying

$$
s=\frac{\varphi_{x x}}{1+\varphi_{x}^{2}}+c \sqrt{1+\varphi_{x}^{2}}
$$

The readers can refer to Fig. 3 in Ninomiya and Taniguchi [43] for the shape of the function $\varphi$. It follows from Ninomiya and Taniguchi [43, Lemma 2.1] that there exist positive constants $\beta_{2}:=s m_{*}, C_{j}(j=2,3,4)$ and $v_{ \pm}$such that

$$
\begin{aligned}
& \max \left\{\left|\varphi^{\prime \prime}(x)\right|,\left|\varphi^{\prime \prime \prime}(x)\right|\right\} \leq C_{2} \operatorname{sech}\left(\beta_{2} x\right), \\
& C_{3} \operatorname{sech}\left(\beta_{2} x\right) \leq \frac{s}{\sqrt{1+\varphi_{x}^{2}}}-c \leq C_{4} \operatorname{sech}\left(\beta_{2} x\right), \\
& m_{*}|x| \leq \varphi(x) \leq m_{*}|x|+M_{*},
\end{aligned}
$$




$$
v_{-} \leq v(x) \leq v_{+}
$$

for all $x \in \mathbb{R}$, where $M_{*}$ is a bounded positive constant and

$$
v(x)=\frac{s\left(\varphi(x)-m_{*}|x|\right)}{s-c \sqrt{1+\varphi_{x}^{2}}} .
$$

We note that $\beta_{2}=s m_{*}=\frac{s \sqrt{s^{2}-c^{2}}}{c}>0$ and that the curvature of $\varphi=\varphi(x)$ is calculated as

$$
\frac{\varphi^{\prime \prime}(x)}{\left(1+\varphi^{\prime 2}(x)\right)^{3 / 2}}=\frac{s}{\sqrt{1+\varphi^{\prime 2}(x)}}-c .
$$

From (2.1) and (2.2), one observes that

$$
\left|\varphi^{\prime}(x)\right| \leq m_{*}, \quad\left|\varphi^{\prime \prime}(x)\right| \leq C_{2} .
$$

Assumption (F) implies that there exists a positive constant $\delta_{1}\left(0<\delta_{1}<\frac{1}{4}\right)$ with

$$
-f^{\prime}(r) \geq \omega \quad \text { for } r<\delta_{1} \text { or } r>1-\delta_{1}
$$

where

$$
\omega:=\frac{1}{2} \min \left\{-f^{\prime}(0),-f^{\prime}(1)\right\}>0
$$

Since $U(X)$ is increasing in $X \in \mathbb{R}$, we define that positive constants $A$ and $B$ are large enough satisfying

$$
U(-A) \leq \frac{\delta_{1}}{2}, \quad U(B) \geq 1-\frac{\delta_{1}}{2}
$$

respectively. Then, if

$$
\frac{\delta_{1}}{2} \leq U(X) \leq 1-\frac{\delta_{1}}{2}
$$

we have that $-A \leq X \leq B$. Furthermore, it follows from assumption (G) that there exist positive constants $l_{1}$ and $l_{2}$ such that

$$
\left|g^{\prime}(r)\right| \leq l_{1}, \quad\left|g^{\prime \prime}(r)\right| \leq l_{2} \quad \text { for all } r \in[-1,2]
$$

Now, we give the definitions of supersolution and subsolution of (1.9).

Definition 2.1 A function $\bar{u}(x, z, t) \in C^{2,1}\left(\mathbb{R}^{2} \times(0, \infty)\right)$ is called a supersolution of (1.9) if

$$
\bar{u}_{t}-\bar{u}_{x x}-\bar{u}_{z z}+\left(s+g^{\prime}(\bar{u})\right) \bar{u}_{z}-f(\bar{u}) \geq 0, \quad(x, z) \in \mathbb{R}^{2}, t>0 .
$$

Similarly, we can define a subsolution $\underline{u}(x, z, t)$ by reversing the inequality in $(2.7)$.

The next lemma gives a supersolution of (1.9). 
Lemma 2.2 There exist a positive constant $\varepsilon_{0}^{+}$and a positive function $\alpha_{0}^{+}(\varepsilon)$ such that, for $0<\varepsilon<\varepsilon_{0}^{+} \leq 1$ and $0<\alpha \leq \alpha_{0}^{+}(\varepsilon) \leq 1$, the function

$$
v^{+}(x, z ; \varepsilon, \alpha):=U\left(\frac{z+\varphi(\alpha x) / \alpha}{\sqrt{1+\varphi^{\prime 2}(\alpha x)}}\right)+\varepsilon \operatorname{sech}\left(\beta_{2} \alpha x\right)
$$

is a supersolution of (1.9) with

$$
\begin{aligned}
& \lim _{R \rightarrow \infty} \sup _{x^{2}+z^{2} \geq R^{2}}\left|v^{+}(x, z ; \varepsilon, \alpha)-v^{-}(x, z)\right| \leq 2 \varepsilon \\
& v^{-}(x, z)<v^{+}(x, z ; \varepsilon, \alpha) \quad \text { for }(x, z) \in \mathbb{R}^{2} \\
& v_{z}^{+}(x, z ; \varepsilon, \alpha)>0 \quad \text { for }(x, z) \in \mathbb{R}^{2} .
\end{aligned}
$$

Proof Set $\xi:=\alpha x, \sigma(\xi):=\varepsilon \operatorname{sech}\left(\beta_{2} \xi\right)$ and

$$
\zeta:=\frac{z+\varphi(\alpha x) / \alpha}{\sqrt{1+\varphi^{\prime 2}(\alpha x)}}
$$

where $\varepsilon>0$ will be chosen later. A direct calculation yields (see also Ninomiya and Taniguchi [43])

$$
\begin{aligned}
& \zeta_{x}=-\frac{\alpha \varphi^{\prime} \varphi^{\prime \prime}}{1+\varphi^{\prime 2}} \zeta+\frac{\varphi^{\prime}}{\sqrt{1+\varphi^{\prime 2}}} \\
& \zeta_{x x}=-\frac{\alpha^{2}\left(\varphi^{\prime \prime 2}+\varphi^{\prime} \varphi^{\prime \prime \prime}\right)}{1+\varphi^{\prime 2}} \zeta+\frac{3 \alpha^{2} \varphi^{\prime 2} \varphi^{\prime \prime 2}}{\left(1+\varphi^{\prime 2}\right)^{2}} \zeta+\frac{\alpha\left(1-\varphi^{\prime 2}\right) \varphi^{\prime \prime}}{\left(1+\varphi^{\prime 2}\right)^{3 / 2}} .
\end{aligned}
$$

Note that $v^{+}(x, z ; \varepsilon, \alpha)=U(\zeta)+\sigma(\xi)$ and $0 \leq v^{+}(x, z ; \varepsilon, \alpha) \leq 2$. Using (1.7), we have

$$
\begin{aligned}
\mathcal{L}\left[v^{+}\right]= & -v_{x x}^{+}-v_{z z}^{+}+\left(s+g^{\prime}\left(v^{+}\right)\right) v_{z}^{+}-f\left(v^{+}\right) \\
= & -\frac{1}{1+\varphi^{\prime 2}(\xi)} U^{\prime \prime}(\zeta)-\left(U^{\prime}(\zeta) \zeta_{x}\right)_{x}+\left[s+g^{\prime}(U(\zeta)+\sigma(\xi))\right] \frac{1}{\sqrt{1+\varphi^{\prime 2}(\xi)}} U^{\prime}(\zeta) \\
& -f(U(\zeta)+\sigma(\xi))-\alpha^{2} \sigma^{\prime \prime}(\xi) \\
= & \left(1-\frac{1}{1+\varphi^{\prime 2}(\xi)}-\zeta_{x}^{2}\right) U^{\prime \prime}(\zeta)-\zeta_{x x} U^{\prime}(\zeta) \\
& +\frac{1}{s}\left(s+g^{\prime}(U(\zeta))\right)\left(\frac{s}{\sqrt{1+\varphi^{\prime 2}(\xi)}}-c\right) U^{\prime}(\zeta) \\
& +\frac{1}{\sqrt{1+\varphi^{\prime 2}(\xi)}}\left(g^{\prime}(U(\zeta)+\sigma(\xi))-g^{\prime}(U(\zeta))\right) U^{\prime}(\zeta) \\
& +f(U(\zeta))-f(U(\zeta)+\sigma(\xi))-\alpha^{2} \sigma^{\prime \prime}(\xi) \\
= & I_{1}+I_{2}+I_{3}+I_{4}+I_{5},
\end{aligned}
$$

where

$$
I_{1}:=\left(1-\frac{1}{1+\varphi^{\prime 2}(\xi)}-\zeta_{x}^{2}\right) U^{\prime \prime}(\zeta)=-\alpha\left(\left(\frac{\varphi^{\prime} \varphi^{\prime \prime}}{1+\varphi^{\prime 2}}\right)^{2} \alpha \zeta^{2}-\frac{2 \varphi^{\prime 2} \varphi^{\prime \prime}}{\left(1+\varphi^{\prime 2}\right)^{3 / 2}} \zeta\right) U^{\prime \prime}(\zeta)
$$




$$
\begin{aligned}
I_{2} & :=-\zeta_{x x} U^{\prime}(\zeta)=\alpha\left(\frac{\varphi^{\prime \prime 2}+\varphi^{\prime} \varphi^{\prime \prime \prime}}{1+\varphi^{\prime 2}} \alpha \zeta-\frac{3 \varphi^{\prime 2} \varphi^{\prime 2}}{\left(1+\varphi^{\prime 2}\right)^{2}} \alpha \zeta+\frac{\left(\varphi^{\prime 2}-1\right) \varphi^{\prime \prime}}{\left(1+\varphi^{\prime 2}\right)^{3 / 2}} \zeta\right) U^{\prime}(\zeta), \\
I_{3} & :=\frac{1}{s}\left(s+g^{\prime}(U(\zeta))\right)\left(\frac{s}{\sqrt{1+\varphi^{\prime 2}(\xi)}}-c\right) U^{\prime}(\zeta), \\
I_{4} & :=\frac{1}{\sqrt{1+\varphi^{\prime 2}(\xi)}}\left(g^{\prime}(U(\zeta)+\sigma(\xi))-g^{\prime}(U(\zeta))\right) U^{\prime}(\zeta) \\
& =\frac{1}{\sqrt{1+\varphi^{\prime 2}(\xi)}} g^{\prime \prime}\left(U(\zeta)+\vartheta_{0} \sigma(\xi)\right) U^{\prime}(\zeta) \sigma(\xi), \\
I_{5} & :=f(U(\zeta))-f(U(\zeta)+\sigma(\xi))-\alpha^{2} \sigma^{\prime \prime}(\xi),
\end{aligned}
$$

where $0<\vartheta_{0}<1$. By (1.8) and (2.1), we can easily show that

$$
\left|I_{1}\right| \leq C_{5} \alpha \operatorname{sech}\left(\beta_{2} \xi\right), \quad\left|I_{2}\right| \leq C_{6} \alpha \operatorname{sech}\left(\beta_{2} \xi\right)
$$

for $0<\alpha \leq 1$. From (2.5), we have

$$
\sqrt{1+\varphi^{\prime 2}}<s / c
$$

By assumption (C), we have

$$
\epsilon:=s+\min _{r \in[0,1]} g^{\prime}(r)>0
$$

Following from (2.2), (2.11), and (2.12), we have

$$
I_{3} \geq \frac{\epsilon}{s}\left(\frac{s}{\sqrt{1+\varphi^{\prime 2}(\xi)}}-c\right) U^{\prime}(\zeta) \geq \frac{\epsilon}{s} C_{3} \operatorname{sech}\left(\beta_{2} \xi\right) U^{\prime}(\zeta)=C_{7} \operatorname{sech}\left(\beta_{2} \xi\right) U^{\prime}(\zeta)>0,
$$

where $C_{7}=\frac{\epsilon}{s} C_{3}>0$. Letting

$$
0<\varepsilon<\varepsilon_{0}^{+} \leq \frac{\delta_{1}}{2},
$$

it follows that

$$
0<\sigma(\xi)<\frac{\delta_{1}}{2} .
$$

If $U(\zeta) \leq U(-A) \leq \frac{\delta_{1}}{2}$ or $U(\zeta) \geq U(B) \geq 1-\frac{\delta_{1}}{2}$, then $\zeta \leq-A$ or $\zeta \geq B$. By (2.14), we have that $U(\zeta)+\vartheta \sigma(\xi)<\delta_{1}$ or $U(\zeta)+\vartheta \sigma(\xi)>1-\frac{\delta_{1}}{2}>1-\delta_{1}$, where $0<\vartheta<1$. Then

$$
\begin{aligned}
I_{5} & =-f^{\prime}(U(\zeta)+\vartheta \sigma(\xi)) \sigma(\xi)-\alpha^{2} \sigma^{\prime \prime}(\xi) \\
& \geq \omega \sigma(\xi)-C_{8} \alpha^{2} \varepsilon \operatorname{sech}\left(\beta_{2} \xi\right)=\left(\omega-C_{8} \alpha^{2}\right) \varepsilon \operatorname{sech}\left(\beta_{2} \xi\right) .
\end{aligned}
$$

From (1.8), we have

$$
\begin{aligned}
\left|I_{4}\right| & =\frac{1}{\sqrt{1+\varphi^{\prime 2}(\xi)}}\left|g^{\prime \prime}\left(U(\zeta)+\theta_{0} \sigma(\xi)\right)\right| U^{\prime}(\zeta) \varepsilon \operatorname{sech}\left(\beta_{2} \xi\right) \\
& \leq l_{2} C_{1} \varepsilon \operatorname{sech}\left(\beta_{2} \xi\right) e^{-\beta_{1}|\zeta|}
\end{aligned}
$$


where $l_{2}$ is defined in (2.6). Since $\zeta \leq-A$ or $\zeta \geq B$, we can take $A$ and $B$ large enough such that

$$
\min \{A, B\}>\max \left\{1, \frac{1}{\beta_{1}} \ln \frac{2 l_{2} C_{1}}{\omega}\right\},
$$

then we have

$$
\left|I_{4}\right| \leq \frac{\omega}{2} \varepsilon \operatorname{sech}\left(\beta_{2} \xi\right) .
$$

It follows that

$$
\begin{aligned}
\mathcal{L}\left[v^{+}\right] & \geq\left(\omega-C_{8} \alpha^{2}\right) \varepsilon \operatorname{sech}\left(\beta_{2} \xi\right)-\frac{\omega}{2} \varepsilon \operatorname{sech}\left(\beta_{2} \xi\right)-\left(C_{5}+C_{6}\right) \alpha \operatorname{sech}\left(\beta_{2} \xi\right) \\
& =\left(\frac{\omega}{2}-C_{8} \alpha^{2}\right) \varepsilon \operatorname{sech}\left(\beta_{2} \xi\right)-\left(C_{5}+C_{6}\right) \alpha \operatorname{sech}\left(\beta_{2} \xi\right) \\
& \geq \frac{\omega}{4} \varepsilon \operatorname{sech}\left(\beta_{2} \xi\right)>0
\end{aligned}
$$

provided that

$$
0<\alpha \leq \min \left\{1, \sqrt{\frac{\omega}{8 C_{8}}}, \frac{\omega}{8\left(C_{5}+C_{6}\right)} \varepsilon\right\} .
$$

If $U(-A) \leq U(\zeta) \leq U(B)$, namely $-A \leq \zeta \leq B$, then we have

$$
\begin{aligned}
& I_{3} \geq C_{7} U^{\prime}(\zeta) \operatorname{sech}\left(\beta_{2} \xi\right) \geq C_{7} q \operatorname{sech}\left(\beta_{2} \xi\right) \\
& \left|I_{5}\right| \leq C_{9} \varepsilon \operatorname{sech}\left(\beta_{2} \xi\right)+C_{8} \alpha^{2} \varepsilon \operatorname{sech}\left(\beta_{2} \xi\right) \leq\left(C_{9}+C_{8} \alpha\right) \varepsilon \operatorname{sech}\left(\beta_{2} \xi\right)
\end{aligned}
$$

where

$$
q:=\min _{-A \leq \zeta \leq B} U^{\prime}(\zeta)>0
$$

Moreover, from (2.15), we have $\left|I_{4}\right| \leq l_{2} C_{1} \varepsilon \operatorname{sech}\left(\beta_{2} \xi\right)$. Eventually, we have

$$
\begin{aligned}
\mathcal{L}\left[v^{+}\right] & \geq C_{7} q \operatorname{sech}\left(\beta_{2} \xi\right)-\left(C_{5}+C_{6}+C_{8} \varepsilon\right) \alpha \operatorname{sech}\left(\beta_{2} \xi\right)-\left(l_{2} C_{1}+C_{9}\right) \varepsilon \operatorname{sech}\left(\beta_{2} \xi\right) \\
& \geq \frac{1}{2} C_{7} q \operatorname{sech}\left(\beta_{2} \xi\right)>0
\end{aligned}
$$

if

$$
0<\varepsilon \leq \min \left\{1, \frac{\delta_{1}}{2}, \frac{C_{7} q}{4\left(l_{2} C_{1}+C_{9}\right)}\right\}
$$

and

$$
0<\alpha \leq \min \left\{1, \frac{C_{7} q}{4\left(C_{5}+C_{6}+\varepsilon C_{8}\right)}\right\} .
$$


Take $\varepsilon$ and $\alpha$ satisfying (2.13), (2.16), (2.18), and (2.19), then we have

$$
\mathcal{L}\left[v^{+}\right] \geq \frac{1}{4} \min \left\{\omega \varepsilon, 2 C_{7} q\right\} \operatorname{sech}\left(\beta_{2} \xi\right)>0 \quad \text { in } \mathbb{R}^{2} .
$$

Thus we proved that $v^{+}$is a supersolution.

Furthermore, if we take $\alpha<\frac{\varepsilon e^{2} c^{2} \beta_{1}^{2} v_{-}}{4 C_{1} C_{4} s}$, where $e$ is the exponential, we can prove (2.9) by an argument similar to inequality (2.3) of Ninomiya and Taniguchi [43] and (2.7) of Wang and Wu [60]. The proof of (2.8) is similar to (2.2) of Ninomiya and Taniguchi [43] and (2.6) of Wang and Wu [60], we omit the details. In addition, (2.10) immediately follows from the definition of $v^{+}$.

Take

$$
\varepsilon_{0}^{+}:=\min \left\{1, \frac{\delta_{1}}{2}, \frac{C_{7} q}{4\left(l_{2} C_{1}+C_{9}\right)}\right\}
$$

and

$$
\alpha_{0}^{+}:=\min \left\{1, \sqrt{\frac{\omega}{8 C_{8}}}, \frac{\omega}{8\left(C_{5}+C_{6}\right)} \varepsilon, \frac{C_{7} q}{4\left(C_{5}+C_{6}+\varepsilon C_{8}\right)}, \frac{\varepsilon e^{2} c^{2} \beta_{1}^{2} v_{-}}{4 C_{1} C_{4} s}\right\} .
$$

It follows that (2.8)-(2.10) hold for $(x, z) \in \mathbb{R}^{2}$ if $0<\varepsilon<\varepsilon_{0}^{+}$and $0<\alpha<\alpha_{0}^{+}(\varepsilon)$. This completes the proof.

In the following, we give the existence of traveling curved fronts of (1.1).

Theorem 2.3 There exists a traveling wave solution $u(x, y, t)=v_{*}(x, y+s t)$ of $(1.1)$ satisfying (1.10) and

$$
\begin{aligned}
& \lim _{R \rightarrow \infty} \sup _{x^{2}+z^{2} \geq R^{2}}\left|v_{*}(x, z)-v^{-}(x, z)\right|=0, \\
& v^{-}(x, z)<v_{*}(x, z)<\min \left\{1, v^{+}(x, z ; \varepsilon, \alpha\}, \quad \forall(x, z) \in \mathbb{R}^{2},\right. \\
& v_{*}(x, z)=v_{*}(-x, z), \quad \forall(x, z) \in \mathbb{R}^{2}, \\
& \frac{\partial}{\partial z} v_{*}(x, z)>0, \quad \forall(x, z) \in \mathbb{R}^{2}, \\
& \frac{\partial}{\partial x} \nu_{*}(x, z)>0, \quad \forall(x, z) \in(0, \infty) \times \mathbb{R} .
\end{aligned}
$$

Proof To establish a traveling curved front of (1.1), we first construct a classical solution $v_{*}$ of the stationary equation (1.10).

Let

$$
N:=\sup _{r \in[-1,2]}\left|f^{\prime}(r)\right|+C_{1} l_{2}
$$

where $C_{1}$ and $l_{2}$ are as in (1.8) and (2.6), respectively. Consider the following linear initial value problem:

$$
\left\{\begin{array}{l}
u_{t}-u_{x x}-u_{z z}+\left(s+g^{\prime}\left(v^{-}(x, z)\right)\right) u_{z}+N u=N v^{-}(x, z)+f\left(v^{-}(x, z)\right) \\
u(x, z, 0)=v^{-}(x, z)
\end{array}\right.
$$


where $(x, z) \in \mathbb{R}^{2}, t \geq 0$. By Lunardi [35, Theorem 5.1.3], there exists a smooth solution

$$
u\left(x, z, t ; v^{-}\right) \in C^{2+\alpha, 1+\frac{\alpha}{2}}\left(\mathbb{R}^{2} \times(0,+\infty)\right) \cap C\left(\mathbb{R}^{2} \times[0,+\infty)\right)
$$

of problem (2.20). Furthermore, since $g^{\prime}\left(v^{-}(x, z)\right), f\left(v^{-}(x, z)\right) \in C^{\alpha}\left(\mathbb{R}^{2}\right)$, by Lunardi [35, Theorem 5.1.4 (iv)], there exists a constant $C>0$ such that

$$
\left\|u\left(\cdot, \cdot, t ; v^{-}\right)\right\|_{C^{2+\alpha\left(\mathbb{R}^{2}\right)}} \leq C, \quad \forall t>1
$$

Since $v^{-}(x, z)=v^{-}(-x, z)$, then $u\left(x, z, t ; v^{-}\right)=u\left(-x, z, t ; v^{-}\right)$for $(x, z) \in \mathbb{R}^{2}$ and $t \geq 0$. Therefore, we have $\left.\frac{\partial}{\partial x} u\left(x, z, t ; v^{-}\right)\right|_{x=0}=0$. In addition, similar to Wang [55, Corollary 2.8] we can prove that $\frac{\partial}{\partial x} u\left(-x, z, t ; v^{-}\right)>0$ for $(x, z) \in(0, \infty) \times \mathbb{R}$ and $t>0$.

Let $\phi^{+}(x, z)=U\left(\frac{c}{s}\left(z+m_{*} x\right)\right)$ and $\phi^{-}(x, z)=U\left(\frac{c}{s}\left(z-m_{*} x\right)\right), \forall(x, z) \in \mathbb{R}^{2}$. Let $\Omega=(0, \infty) \times \mathbb{R}$. Then we have

$$
\begin{aligned}
& -\phi_{x x}^{+}-\phi_{z z}^{+}+\left(s+g^{\prime}\left(v^{-}(x, z)\right)\right) \phi_{z}^{+}+N \phi^{+}-N v^{-}(x, z)-f\left(v^{-}(x, z)\right) \\
& =-\phi_{x x}^{+}-\phi_{z z}^{+}+\left(s+g^{\prime}\left(\phi^{+}(x, z)\right)\right) \phi_{z}^{+}+N \phi^{+}-N \phi^{+}(x, z)-f\left(\phi^{+}(x, z)\right)=0
\end{aligned}
$$

for any $(x, z) \in \Omega$. Furthermore $\frac{\partial}{\partial n} \phi^{+}(x, z)=-\frac{c}{s} m_{*} U^{\prime}\left(\frac{c}{s}\left(z+m_{*} x\right)\right)<0$ on $\partial \Omega$, where $\frac{\partial}{\partial n}$ is the outward normal derivative on $\partial \Omega$.

Let $u^{+}=u-\phi^{+}$, then $u^{+}$satisfies the following inequalities:

$$
\left\{\begin{array}{l}
u_{t}^{+}-u_{x x}^{+}-u_{z z}^{+}+\left(s+g^{\prime}\left(v^{-}(x, z)\right)\right) u_{z}^{+}+N u^{+} \geq 0, \quad(x, z, t) \in \Omega \times(0, \infty), \\
u^{+}(x, z, 0)=u(x, z, 0)-\phi^{+}(x, z)=v^{-}(x, z)-\phi^{+}(x, z) \geq 0, \quad(x, z) \in \Omega, \\
\frac{\partial}{\partial n} u^{+}(x, z, t) \geq 0, \quad(x, z, t) \in \partial \Omega \times(0, \infty) .
\end{array}\right.
$$

Using the comparison principle [14, Theorem 25.6], we have $u^{+}(x, z, t) \geq 0$, which implies

$$
u\left(x, z, t ; v^{-}\right) \geq \phi^{+}(x, z), \quad \forall(x, z, t) \in \Omega \times[0, \infty) .
$$

Similarly, if we let $u^{-}=u-\phi^{-}$and $\Omega^{\prime}=(-\infty, 0] \times \mathbb{R}$, we can show that

$$
u\left(x, z, t ; v^{-}\right) \geq \phi^{-}(x, z), \quad \forall(x, z, t) \in \Omega^{\prime} \times[0, \infty) .
$$

It follows that

$$
u\left(x, z, t ; v^{-}\right) \geq v^{-}(x, z), \quad \forall(x, z, t) \in \mathbb{R}^{2} \times[0, \infty) .
$$

On the other hand, since $v^{+}=v^{+}(x, z ; \varepsilon, \alpha)$ is a supersolution of (1.9), we get that

$$
-v_{x x}^{+}-v_{z z}^{+}+\left(s+g^{\prime}\left(v^{+}(x, z ; \varepsilon, \alpha)\right)\right) v_{z}^{+}+N v^{+} \geq N v^{+}(x, z ; \varepsilon, \alpha)+f\left(v^{+}(x, z ; \varepsilon, \alpha)\right) .
$$

Let $v=v^{+}-u$, we have

$$
\begin{aligned}
v_{t} & -v_{x x}-v_{z z}+\left(s+g^{\prime}\left(v^{-}\right)\right) v_{z}+N v \\
& \geq N\left(v^{+}-v^{-}\right)+f\left(v^{+}\right)-f\left(v^{-}\right)-\left(g^{\prime}\left(v^{+}\right)-g^{\prime}\left(v^{-}\right)\right) v_{z}^{+} \geq 0 .
\end{aligned}
$$


From Lemma 2.2, the function $v$ satisfies the following:

$$
\left\{\begin{array}{l}
v_{t}-v_{x x}-v_{z z}+\left(s+g^{\prime}\left(v^{-}(x, z)\right)\right) v_{z}+N v \geq 0 \\
v(x, z, 0)=v^{+}-v^{-}>0
\end{array}\right.
$$

Also, by the comparison principle [14, Theorem 25.6], we get that $v(x, z, t) \geq 0$. Then

$$
v^{+}(x, z ; \varepsilon, \alpha) \geq u\left(x, z, t ; v^{-}\right), \quad \forall(x, z, t) \in \mathbb{R}^{2} \times[0, \infty)
$$

Combining (2.22) and (2.23), we obtain that

$$
v^{-}(x, z) \leq u\left(x, z, t ; v^{-}\right) \leq v^{+}(x, z ; \varepsilon, \alpha), \quad \forall(x, z, t) \in \mathbb{R}^{2} \times[0, \infty) .
$$

Next, we will prove that $u\left(x, z, t ; v^{-}\right)$is monotone increasing with respect to $t \in(0, \infty)$. In fact, from (2.22), we know that, for $\forall \epsilon>0, u\left(\cdot, \cdot, \epsilon ; v^{-}\right)>u\left(\cdot, \cdot, 0 ; v^{-}\right)=v^{-}(\cdot, \cdot)$, the comparison principle [14, Theorem 25.6] implies that

$$
u\left(\cdot, \cdot, t+\epsilon ; v^{-}\right)>u\left(\cdot, \cdot, t ; v^{-}\right)
$$

Then we have proved that $u\left(\cdot, \cdot, t ; v^{-}\right)$is monotone increasing with respect to $t$.

Let us show that $u\left(x, z, t ; v^{-}\right)$is monotone increasing with respect to $z$. Taking the derivative of equation (2.20) with respect to $z$, we have

$$
\left\{\begin{array}{l}
\left(u_{z}\right)_{t}-\left(u_{z}\right)_{x x}-\left(u_{z}\right)_{z z}+s\left(u_{z}\right)_{z}+N u_{z}+g^{\prime \prime}\left(v^{-}(x, z)\right) v_{z}^{-}(x, z) u_{z}+g^{\prime}\left(v^{-}(x, z)\right)\left(u_{z}\right)_{z} \\
\quad=N v_{z}^{-}(x, z)+f^{\prime}\left(v^{-}(x, z)\right) v_{z}^{-}(x, z) \\
u_{z}(x, z, 0)=v_{z}^{-}(x, z)>0
\end{array}\right.
$$

Therefore

$$
\left\{\begin{array}{l}
\left(u_{z}\right)_{t}-\left(u_{z}\right)_{x x}-\left(u_{z}\right)_{z z}+\left(s+g^{\prime}\left(v^{-}(x, z)\right)\right)\left(u_{z}\right)_{z}+\left(N+g^{\prime \prime}\left(v^{-}(x, z)\right) v_{z}^{-}(x, z)\right) u_{z} \geq 0 \\
u_{z}(x, z, 0)=v_{z}^{-}(x, z)>0
\end{array}\right.
$$

Using the comparison principle [14, Theorem 25.6], we have $u_{z}\left(x, z, t ; v^{-}\right)>0$.

As above, we conclude that the $\operatorname{limit}_{t \rightarrow \infty} u\left(x, z, t ; v^{-}\right):=u^{1}(x, z)$ exists. It follows from (2.21) that $u^{1}(x, z) \in C^{2+\alpha}\left(\mathbb{R}^{2}\right)$ and

$$
\left\{\begin{array}{l}
v^{-}(x, z) \leq u^{1}(x, z) \leq v^{+}(x, z ; \varepsilon, \alpha), \quad \forall(x, z) \in \mathbb{R}^{2} \\
u^{1}(x, z)=u^{1}(-x, z), \quad \forall(x, z) \in \mathbb{R}^{2} \\
u_{z}^{1}(x, z) \geq 0, \quad \forall(x, z) \in \mathbb{R}^{2} \\
u_{x}^{1}(x, z) \geq 0, \quad \forall(x, z) \in(0, \infty) \times \mathbb{R}
\end{array}\right.
$$

Now we show that $u^{1}$ further satisfies

$$
-u_{x x}^{1}-u_{z z}^{1}+\left(s+g^{\prime}\left(v^{-}(x, z)\right)\right) u_{z}^{1}+N u^{1}=N v^{-}(x, z)+f\left(v^{-}(x, z)\right), \quad \forall(x, z) \in \mathbb{R}^{2}
$$


Let $\phi \in C_{0}^{\infty}\left(\mathbb{R}^{2}\right)$. Since $g^{\prime}\left(v^{-}(x, z)\right)$ is differentiable on $z \in \mathbb{R}$, we have

$$
\begin{gathered}
\int_{\mathbb{R}^{2}} \frac{\partial}{\partial t} u \phi \mathrm{d} z \mathrm{~d} x-\int_{\mathbb{R}^{2}} u \Delta \phi \mathrm{d} z \mathrm{~d} x-\int_{\mathbb{R}^{2}} u \frac{\partial}{\partial z}\left(\left(s+g^{\prime}\left(v^{-}\right)\right) \phi\right) \mathrm{d} z \mathrm{~d} x+N \int_{\mathbb{R}^{2}} u \phi \mathrm{d} z \mathrm{~d} x \\
=\int_{\mathbb{R}^{2}}\left(N v^{-}(x, z)+f\left(v^{-}(x, z)\right)\right) \phi(x, z) \mathrm{d} z \mathrm{~d} x .
\end{gathered}
$$

For $T>0$, multiplying both sides of the aforementioned equality by $\frac{1}{T}$ and integrating over $(T, 2 T)$, we obtain

$$
\begin{aligned}
& \int_{\mathbb{R}^{2}} \frac{u(x, z, 2 T)-u(x, z, T)}{T} \phi \mathrm{d} z \mathrm{~d} x-\int_{\mathbb{R}^{2}} \frac{1}{T} \int_{T}^{2 T} u \mathrm{~d} t \Delta \phi \mathrm{d} z \mathrm{~d} x \\
& \quad-\int_{\mathbb{R}^{2}} \frac{1}{T} \int_{T}^{2 T} u \mathrm{~d} t \frac{\partial}{\partial z}\left(\left(s+g^{\prime}\left(v^{-}\right)\right) \phi\right) \mathrm{d} z \mathrm{~d} x+N \int_{\mathbb{R}^{2}} \frac{1}{T} \int_{T}^{2 T} u \mathrm{~d} t \phi \mathrm{d} z \mathrm{~d} x \\
&=\int_{\mathbb{R}^{2}}\left(N v^{-}(x, z)+f\left(v^{-}(x, z)\right)\right) \phi(x, z) \mathrm{d} z \mathrm{~d} x .
\end{aligned}
$$

Letting $T \rightarrow+\infty$ yields

$$
\begin{aligned}
& -\int_{\mathbb{R}^{2}} u^{1} \Delta \phi \mathrm{d} z \mathrm{~d} x-\int_{\mathbb{R}^{2}} u^{1} \frac{\partial}{\partial z}\left(\left(s+g^{\prime}\left(v^{-}\right)\right) \phi\right) \mathrm{d} z \mathrm{~d} x+N \int_{\mathbb{R}^{2}} u^{1} \phi \mathrm{d} z \mathrm{~d} x \\
& \quad=\int_{\mathbb{R}^{2}}\left(N v^{-}(x, z)+f\left(v^{-}(x, z)\right)\right) \phi(x, z) \mathrm{d} z \mathrm{~d} x,
\end{aligned}
$$

which implies that

$$
\int_{\mathbb{R}^{2}}\left(-\Delta u^{1}+\left(s+g^{\prime}\left(v^{-}\right) u_{z}^{1}+N u^{1}-N v^{1}-f\left(v^{-}\right)\right) \phi \mathrm{d} z \mathrm{~d} x=0\right.
$$

Due to the arbitrariness of $\phi \in C_{0}^{\infty}\left(\mathbb{R}^{2}\right)$, we conclude that equality (2.24) holds.

By virtue of assumption $(\mathrm{G})$ and the definition of $N$, we have

$$
\begin{aligned}
& -u_{x x}^{1}-u_{z z}^{1}+\left(s+g^{\prime}\left(u^{1}\right)\right) u_{z}^{1}+N u^{1} \\
& \quad=\left(g^{\prime}\left(u^{1}\right)-g^{\prime}\left(v^{-}\right)\right) u_{z}^{1}+N v^{-}+f\left(v^{-}\right) \leq N u^{1}+f\left(u^{1}\right), \quad(x, z) \in \mathbb{R}^{2} .
\end{aligned}
$$

By (2.24) and (2.25), we know that $u^{1}(x, z)$ is a subsolution of the following problem:

$$
\left\{\begin{array}{l}
w_{t}-w_{x x}-w_{z z}+\left(s+g^{\prime}(w)\right) w_{z}-f(w)=0, \quad(x, z) \in \mathbb{R}^{2}, t>0, \\
w(x, z, 0)=u^{1}(x, z), \quad(x, z) \in \mathbb{R}^{2} .
\end{array}\right.
$$

The local existence of a unique solution $w\left(x, z, t ; u^{1}\right)$ of the last equation follows from [35, Theorem 7.1.2, Propositions 7.1.9 and 7.1.10, and Remark 7.1.12], see also [8, Proposition A.3]. Since $u^{1}(x, z)$ and $v^{+}(x, z ; \varepsilon, \alpha)$ are sub- and supersolutions of the last equation respectively, we have that the unique solution $w\left(x, z, t ; u^{1}\right)$ exists globally. It follows from [34, Chapter V, Theorem 3.1; Chapter VII, Theorem 5.1] that there exists $K>0$ such that

$$
\left\|w\left(\cdot, t ; u^{1}\right)\right\|_{C^{2}\left(\mathbb{R}^{2}\right)} \leq K, \quad \forall t \geq 1 .
$$


Consequently, there exists $K^{\prime}>0$ such that

$$
\left\|w\left(x, z, \cdot ; u^{1}\right)\right\|_{C^{1}([1, \infty))} \leq K^{\prime}, \quad \forall(x, z) \in \mathbb{R}^{2} .
$$

Now by [35, Theorem 5.1.4] there exists a constant $C>0$ such that

$$
\left\|w\left(\cdot, t ; u^{1}\right)\right\|_{C^{2+\alpha}\left(\mathbb{R}^{2}\right)} \leq C, \quad \forall t>2 .
$$

By the arguments similar to those for $u\left(x, z, t ; v^{-}\right)$and $u^{1}(x, z)$, we have that $w\left(x, z, t ; u^{1}\right)$ is monotone increasing in $t>0$ and the limit function

$$
v_{*}(x, z):=\lim _{t \rightarrow \infty} w\left(x, z, t ; u^{1}\right)
$$

exists. In particular, $v_{*}(x, z)$ satisfies $\left\|v_{*}(\cdot)\right\|_{C^{2+\alpha}\left(\mathbb{R}^{2}\right)} \leq C$ with some constant $C>0$ and

$$
\begin{aligned}
& \mathcal{L}\left[\nu_{*}\right]=0, \quad \nu_{*}(-x, z)=v_{*}(x, z), \quad \text { and } \quad \frac{\partial}{\partial z} \nu_{*}(x, z)>0, \quad \forall(x, z) \in \mathbb{R}^{2}, \\
& \frac{\partial}{\partial x} \nu_{*}(x, z)>0, \quad \forall(x, z) \in(0, \infty) \times \mathbb{R}, \\
& v^{-}(x, z) \leq v_{*}(x, z) \leq v^{+}(x, z ; \varepsilon, \alpha), \quad \forall(x, z) \in \mathbb{R}^{2} .
\end{aligned}
$$

Since $\varepsilon \in\left(0, \varepsilon_{0}^{+}\right)$and $\alpha \in\left(0, \alpha_{0}^{+}\right)$are arbitrary, it follows from (2.8) that

$$
\lim _{R \rightarrow \infty} \sup _{x^{2}+z^{2} \geq R^{2}}\left|v_{*}(x, z)-v^{-}(x, z)\right|=0
$$

In addition, it is clear that $v_{*}(x, z)<1$ for any $(x, z) \in \mathbb{R}^{2}$. This completes the proof.

\section{Global asymptotic stability}

In this section we develop the arguments of Ninomiya and Taniguchi [43] to establish the stability of traveling curved front $v_{*}$ obtained in Sect. 2. We prove that (1.14) holds true for $u_{0}(x, z) \geq v^{-}(x, z)$. See Theorem 3.6. Consider the following initial value problem:

$$
\left\{\begin{array}{l}
w_{t}-w_{x x}-w_{z z}+\left(s+g^{\prime}(w)\right) w_{z}-f(w)=0, \quad(x, z) \in \mathbb{R}^{2}, t>0, \\
w(x, z, 0)=u_{0}(x, z), \quad(x, z) \in \mathbb{R}^{2},
\end{array}\right.
$$

where $u_{0} \in B U C^{1}\left(\mathbb{R}^{2}\right)$ is a given initial function. The global existence of a unique solution $w\left(x, z, t ; u_{0}\right)$ of equation (3.1) follows from [35, Theorem 7.1.2, Propositions 7.1.9 and 7.1.10, and Remark 7.1.12] and assumptions (F) and (G), see also [8, Proposition A.3 and Theorem A.7]. In particular, $w\left(t ; u_{0}\right)(\cdot) \in C^{1}\left((0, \infty), B U C\left(\mathbb{R}^{2}\right)\right) \cap C\left((0, \infty), B U C^{2}\left(\mathbb{R}^{2}\right)\right) \cap$ $C\left([0, \infty), B U C^{1}\left(\mathbb{R}^{2}\right)\right)$, where $w\left(t ; u_{0}\right)(x, z):=w\left(x, z, t ; u_{0}\right)$. It follows from [34, Chapter $\mathrm{V}$, Theorem 3.1] that there exists a constant $K\left(u_{0}\right)>0$ such that

$$
\left\|w\left(\cdot, t ; u_{0}\right)\right\|_{C^{1}\left(\mathbb{R}^{2}\right)}<K\left(u_{0}\right), \quad t \geq 0 .
$$

Using [34, Chapter VII, Theorem 5.1], we further have that there exists $K^{\prime}\left(u_{0}\right)>0$ such that $\left\|w\left(\cdot, t ; u_{0}\right)\right\|_{C^{2}\left(\mathbb{R}^{2}\right)} \leq K^{\prime}\left(u_{0}\right)$ for any $t \geq 1$ and $\left\|w\left(x, z, \cdot ; u_{0}\right)\right\|_{C^{1}([1, \infty))} \leq K^{\prime}\left(u_{0}\right)$ for any $(x, z) \in \mathbb{R}^{2}$. 
Let $w_{1}(t)$ be defined by

$$
\left\{\begin{array}{l}
w_{1}^{\prime}(t)=f\left(w_{1}(t)\right) \text { for } t>0, \\
w_{1}(0)=\min \left\{0, \inf _{(x, z) \in \mathbb{R}^{2}} u_{0}(x, z)\right\} \leq 0,
\end{array}\right.
$$

and $w_{2}(t)$ be defined by

$$
\left\{\begin{array}{l}
w_{2}^{\prime}(t)=f\left(w_{2}(t)\right) \quad \text { for } t>0 \\
w_{2}(0)=\max \left\{1, \sup _{(x, z) \in \mathbb{R}^{2}} u_{0}(x, z)\right\} \geq 1
\end{array}\right.
$$

Then $w_{1}(t)$ and $w_{2}(t)$ are solutions of (3.1) with $w_{1}(0) \leq u_{0}(x, z) \leq w_{2}(0)$. The comparison principle [14, Theorem 25.6] implies

$$
w_{1}(t) \leq w\left(x, z, t ; u_{0}\right) \leq w_{2}(t) \quad \text { for }(x, z) \in \mathbb{R}^{2}, t>0 .
$$

Since $\lim _{t \rightarrow \infty} w_{1}(t)=0$ and $\lim _{t \rightarrow \infty} w_{2}(t)=1$, then we have

$$
0 \leq \liminf _{t \rightarrow \infty} w\left(x, z, t ; u_{0}\right) \leq \limsup _{t \rightarrow \infty} w\left(x, z, t ; u_{0}\right) \leq 1 \quad \text { for }(x, z) \in \mathbb{R}^{2} .
$$

The following theorem shows the continuous dependence of solutions of (3.1) on initial values.

Lemma 3.1 Let $w^{(j)}(x, z, t)$ be the solution of

$$
\left\{\begin{array}{l}
w_{t}^{(j)}+\mathcal{L}\left[w^{(j)}\right]=0 \quad \text { for }(x, z) \in \mathbb{R}^{2}, t>0 \\
w^{(j)}(x, z, 0)=w_{0}^{(j)}(x, z) \quad \text { for }(x, z) \in \mathbb{R}^{2}
\end{array}\right.
$$

where $j=1,2$. Assume that $w_{0}^{(j)}(x, z) \in B U C^{1}\left(\mathbb{R}^{2}\right)(j=1,2)$ and

$$
-1 \leq w_{0}^{(j)}(x, z) \leq 2 \quad \text { for }(x, z) \in \mathbb{R}^{2}, j=1,2,
$$

then there exists a constant $A_{0}>1$ such that

$$
\left\|w^{(2)}(\cdot, t)-w^{(1)}(\cdot, t)\right\|_{C^{1}\left(\mathbb{R}^{2}\right)} \leq A_{0}^{t+1}\left\|w_{0}^{(2)}(\cdot)-w_{0}^{(1)}(\cdot)\right\|_{C^{1}\left(\mathbb{R}^{2}\right)}, \quad t \in[0, \infty) .
$$

Proof Since $-1 \leq w_{0}^{(j)}(x, z) \leq 2$, the comparison principle [14, Theorem 25.6] implies $-1 \leq$ $w^{(j)}(x, z, t) \leq 2$ for any $(x, z) \in \mathbb{R}^{2}$ and $t>0, j=1,2$. It follows from (3.2) that there exists $K^{*}>0$ such that

$$
\left|w_{z}^{(j)}(x, z, t)\right| \leq K^{*}, \quad(x, z) \in \mathbb{R}^{2}, t \in[0, \infty), j=1,2 .
$$

Define $\hat{w}(x, z, t)=w^{(2)}(x, z, t)-w^{(1)}(x, z, t)$ satisfying

$$
\left\{\begin{array}{l}
\hat{w}_{t}-\hat{w}_{x x}-\hat{w}_{z z}+G_{1}(x, z, t) \hat{w}_{z}+G_{2}(x, z, t) \hat{w}=0, \quad(x, z) \in \mathbb{R}^{2}, t>0, \\
\hat{w}(x, z, 0)=w_{0}^{(2)}(x, z)-w_{0}^{(1)}(x, z), \quad(x, z) \in \mathbb{R}^{2},
\end{array}\right.
$$


where

$$
\begin{aligned}
& G_{1}(x, z, t)=s-g^{\prime}\left(w^{(1)}\right), \\
& G_{2}(x, z, t)=g^{\prime \prime}\left(\theta_{1} w^{(2)}+\left(1-\theta_{1}\right) w^{(1)}\right) w_{z}^{(2)}-f^{\prime}\left(\theta_{2} w^{(2)}+\left(1-\theta_{2}\right) w^{(1)}\right) .
\end{aligned}
$$

From (2.6) and (3.4), we have $\left|G_{2}(x, z, t)\right| \leq l_{2} K^{*}+M$, where

$$
M:=\sup _{-1 \leq r \leq 2}\left|f^{\prime}(r)\right|
$$

Since $g \in C^{2+\gamma_{0}}(\mathbb{R})$ and $G_{1}(x, z, t)$ is bounded and continuous in $\mathbb{R}^{2} \times \mathbb{R}^{+}$, Friedman [18, Chapter 1, Theorem 12] implies that the solution $\hat{w}(x, z, t)$ of problem (3.5) can be expressed as

$$
\begin{aligned}
\hat{w}(x, z, t)= & \int_{\mathbb{R}^{2}} \frac{1}{4 \pi t} e^{-\frac{\eta_{1}^{2}+\eta_{2}^{2}}{4 t}} \hat{w}\left(x-\eta_{1}, z-\eta_{2}, 0\right) \mathrm{d} \eta_{1} \mathrm{~d} \eta_{2} \\
& +\int_{0}^{t} \int_{\mathbb{R}^{2}} \frac{1}{4 \pi(t-\tau)} e^{-\frac{\left(x-\eta_{1}\right)^{2}+\left(z-\eta_{2}\right)^{2}}{4(t-\tau)}} \\
& \times\left(G_{1}\left(\eta_{1}, \eta_{2}, \tau\right) \hat{w}_{\eta_{2}}\left(\eta_{1}, \eta_{2}, \tau\right)+G_{2}\left(\eta_{1}, \eta_{2}, \tau\right) \hat{w}\left(\eta_{1}, \eta_{2}, \tau\right)\right) \mathrm{d} \eta_{1} \mathrm{~d} \eta_{2} \mathrm{~d} \tau .
\end{aligned}
$$

Then we have the following estimate:

$$
\|\hat{w}(\cdot, t)\|_{L^{\infty}\left(\mathbb{R}^{2}\right)} \leq\|\hat{w}(\cdot, 0)\|_{L^{\infty}\left(\mathbb{R}^{2}\right)}+K_{1} \int_{0}^{t}\|\hat{w}(\cdot, \tau)\|_{C^{1}\left(\mathbb{R}^{2}\right)} \mathrm{d} \tau
$$

where $K_{1}=s+l_{1}+l_{2} K^{*}+M$. Taking the derivative of function $\hat{w}(x, z, t)$ with respect to $x$, we have

$$
\begin{aligned}
\hat{w}_{x}(x, z, t)= & \int_{\mathbb{R}^{2}} \frac{1}{4 \pi t} e^{-\frac{\eta_{\eta^{2}+\eta_{2}^{2}}^{4 t}}{4} \hat{w}_{x}\left(x-\eta_{1}, z-\eta_{2}, 0\right) \mathrm{d} \eta_{1} \mathrm{~d} \eta_{2}} \\
& +\int_{0}^{t} \int_{\mathbb{R}^{2}} \frac{1}{4 \pi(t-\tau)} e^{-\frac{\left(x-\eta_{1}\right)^{2}+\left(z-\eta_{2}\right)^{2}}{4(t-\tau)}}\left(-\frac{x-\eta_{1}}{2(t-\tau)}\right) \\
& \times\left(G_{1}\left(\eta_{1}, \eta_{2}, \tau\right) \hat{w}_{\eta_{2}}\left(\eta_{1}, \eta_{2}, \tau\right)+G_{2}\left(\eta_{1}, \eta_{2}, \tau\right) \hat{w}\left(\eta_{1}, \eta_{2}, \tau\right)\right) \mathrm{d} \eta_{1} \mathrm{~d} \eta_{2} \mathrm{~d} \tau,
\end{aligned}
$$

and then

$$
\left\|\hat{w}_{x}(\cdot, t)\right\|_{L^{\infty}\left(\mathbb{R}^{2}\right)} \leq\left\|\hat{w}_{x}(\cdot, 0)\right\|_{L^{\infty}\left(\mathbb{R}^{2}\right)}+\frac{K_{1}}{\sqrt{\pi}} \int_{0}^{t}\|\hat{w}(\cdot, \tau)\|_{C^{1}\left(\mathbb{R}^{2}\right)}(t-\tau)^{-\frac{1}{2}} \mathrm{~d} \tau .
$$

Similarly, we have

$$
\left\|\hat{w}_{z}(\cdot, t)\right\|_{L^{\infty}\left(\mathbb{R}^{2}\right)} \leq\left\|\hat{w}_{z}(\cdot, 0)\right\|_{L^{\infty}\left(\mathbb{R}^{2}\right)}+\frac{K_{1}}{\sqrt{\pi}} \int_{0}^{t}\|\hat{w}(\cdot, \tau)\|_{C^{1}\left(\mathbb{R}^{2}\right)}(t-\tau)^{-\frac{1}{2}} \mathrm{~d} \tau
$$

If we set $t \in[0,1]$, since $1 \leq(t-\tau)^{-\frac{1}{2}}$, from (3.7) we have

$$
\|\hat{w}(\cdot, t)\|_{L^{\infty}\left(\mathbb{R}^{2}\right)} \leq\|\hat{w}(\cdot, 0)\|_{L^{\infty}\left(\mathbb{R}^{2}\right)}+K_{1} \int_{0}^{t}\|\hat{w}(\cdot, \tau)\|_{C^{1}\left(\mathbb{R}^{2}\right)}(t-\tau)^{-\frac{1}{2}} \mathrm{~d} \tau .
$$


Combining (3.8), (3.9), and (3.10), we have

$$
\|\hat{w}(\cdot, t)\|_{C^{1}\left(\mathbb{R}^{2}\right)} \leq\|\hat{w}(\cdot, 0)\|_{C^{1}\left(\mathbb{R}^{2}\right)}+\left(1+\frac{2}{\sqrt{\pi}}\right) K_{1} \int_{0}^{t}\|\hat{w}(\cdot, \tau)\|_{C^{1}\left(\mathbb{R}^{2}\right)}(t-\tau)^{-\frac{1}{2}} \mathrm{~d} \tau .
$$

Gronwall's inequality [35, Lemma 7.0.3] implies that there exists a constant $A_{0}>1$, which only depends on $K^{*}>0$, such that

$$
\|\hat{w}(\cdot, t)\|_{C^{1}\left(\mathbb{R}^{2}\right)} \leq A_{0}\|\hat{w}(\cdot, 0)\|_{C^{1}\left(\mathbb{R}^{2}\right)}, \quad t \in[0,1] .
$$

Notice that $w\left(x, z, t+n ; u_{0}\right)=w\left(x, z, t ; w\left(\cdot, n ; u_{0}\right)\right)$ for $(x, z) \in \mathbb{R}^{2}$ and $t>0$, where $n \in \mathbb{N}$. Repeating the above argument, we easily get

$$
\|\hat{w}(\cdot, t)\|_{C^{1}\left(\mathbb{R}^{2}\right)} \leq A_{0}\|\hat{w}(\cdot, n)\|_{C^{1}\left(\mathbb{R}^{2}\right)}, t \in[n, n+1], \quad \forall n \in \mathbb{N}
$$

which implies that

$$
\|\hat{w}(\cdot, t)\|_{C^{1}\left(\mathbb{R}^{2}\right)} \leq A_{0}^{t+1}\|\hat{w}(\cdot, 0)\|_{C^{1}\left(\mathbb{R}^{2}\right)}, \quad t \in[0, \infty)
$$

This completes the proof.

Similar to Ninomiya and Taniguchi [43, Lemma 4.3], we have the following lemma.

Lemma 3.2 There exists a positive constant $\beta_{3}>0$ such that, for $(x, z) \in \mathbb{R}^{2}$, there hold

$$
\begin{array}{ll}
\left(v_{*}\right)_{z}(x, z) \geq \beta_{3} & \text { if } \delta_{1} \leq v_{*}(x, z) \leq 1-\delta_{1}, \\
\left(v^{+}\right)_{z}(x, z) \geq \beta_{3} & \text { if } \delta_{1} \leq v^{+}(x, z) \leq 1-\delta_{1} .
\end{array}
$$

The following two lemmas establish some super- and subsolutions of (3.1).

Lemma 3.3 Let $\bar{v}$ be a supersolution to (1.9) with

$$
\begin{aligned}
& \bar{v}_{z}(x, z)>0,-\delta_{1}<\bar{v}(x, z)<1+\delta_{1} \quad \text { for }(x, z) \in \mathbb{R}^{2}, \\
& \bar{v}_{z}(x, z)>\beta_{3}, \delta_{1} \leq \bar{v}(x, z) \leq 1-\delta_{1} \quad \text { for }(x, z) \in \mathbb{R}^{2} .
\end{aligned}
$$

Let $\underline{v}$ be a subsolution to (1.9) with

$$
\begin{aligned}
& \underline{v}_{z}(x, z)>0,-\delta_{1}<\underline{v}(x, z)<1+\delta_{1} \quad \text { for }(x, z) \in \mathbb{R}^{2}, \\
& \underline{v}_{z}(x, z)>\beta_{3}, \delta_{1} \leq \underline{v}(x, z) \leq 1-\delta_{1} \quad \text { for }(x, z) \in \mathbb{R}^{2},
\end{aligned}
$$

where $\beta_{3}$ and $\delta_{1}$ are defined in Lemma 3.2. Then there exist a large positive constant $\rho$ and a positive constant $\beta$ small enough such that, for any $\delta \in\left(0, \delta_{1} / 2\right], w^{+}$and $w^{-}$defined by

$$
w^{+}(x, z, t ; \bar{v}):=\bar{v}\left(x, z+\rho \delta\left(1-e^{-\beta t}\right)\right)+\delta e^{-\beta t}
$$

and

$$
w^{-}(x, z, t ; \underline{v}):=\underline{v}\left(x, z-\rho \delta\left(1-e^{-\beta t}\right)\right)-\delta e^{-\beta t}
$$

are a supersolution and a subsolution of (3.1), respectively. 
Proof From the definition of $w^{+}$and $w^{-}$, we have

$$
\begin{aligned}
w_{t}^{+}+\mathcal{L}\left[w^{+}\right]= & \delta \beta e^{-\beta t}\left(\rho \bar{v}_{z}-1\right)-\bar{v}_{x x}-\bar{v}_{z z}+\left[s+g^{\prime}\left(\bar{v}+\delta e^{-\beta t}\right)\right] \bar{v}_{z}-f\left(\bar{v}+\delta e^{-\beta t}\right) \\
= & -\bar{v}_{x x}-\bar{v}_{z z}+\left(s+g^{\prime}(\bar{v})\right) \bar{v}_{z}-f(\bar{v})+\delta \beta e^{-\beta t}\left(\rho \bar{v}_{z}-1\right) \\
& +\left(g^{\prime}\left(\bar{v}+\delta e^{-\beta t}\right)-g^{\prime}(\bar{v})\right) \bar{v}_{z}+f(\bar{v})-f\left(\bar{v}+\delta e^{-\beta t}\right) \\
\geq & \delta \beta e^{-\beta t}\left(\rho \bar{v}_{z}-1\right)+\left(g^{\prime}\left(\bar{v}+\delta e^{-\beta t}\right)-g^{\prime}(\bar{v})\right) \bar{v}_{z}+f(\bar{v})-f\left(\bar{v}+\delta e^{-\beta t}\right) \\
= & \delta e^{-\beta t}\left(\left(\rho \beta+\int_{0}^{1} g^{\prime \prime}\left(\bar{v}+\eta \delta e^{-\beta t}\right) \mathrm{d} \eta\right) \bar{v}_{z}-\beta-\int_{0}^{1} f^{\prime}\left(\bar{v}+\eta \delta e^{-\beta t}\right) \mathrm{d} \eta\right)
\end{aligned}
$$

and

$$
\begin{aligned}
w_{t}^{-} & +\mathcal{L}\left[w^{-}\right] \\
& \leq-\delta e^{-\beta t}\left(\left(\rho \beta+\int_{0}^{1} g^{\prime \prime}\left(\underline{v}-\eta \delta e^{-\beta t}\right) \mathrm{d} \eta\right) \underline{v}_{z}-\beta-\int_{0}^{1} f^{\prime}\left(\underline{v}-\eta \delta e^{-\beta t}\right) \mathrm{d} \eta\right),
\end{aligned}
$$

where $\bar{v}=\bar{v}\left(x, z+\rho \delta\left(1-e^{-\beta t}\right)\right)$ and $\underline{v}=\underline{v}\left(x, z-\rho \delta\left(1-e^{-\beta t}\right)\right)$. For convenience, let $v$ be either $\bar{v}$ or $\underline{v}$. By the assumptions, for $\delta_{1} \leq v \leq 1-\delta_{1}$, we have

$$
\begin{aligned}
& \left(\rho \beta+\int_{0}^{1} g^{\prime \prime}\left(v \pm \eta \delta e^{-\beta t}\right) \mathrm{d} \eta\right) v_{z}-\beta-\int_{0}^{1} f^{\prime}\left(v \pm \eta \delta e^{-\beta t}\right) \mathrm{d} \eta \\
& \quad \geq\left(\rho \beta-l_{2}\right) v_{z}-\beta-M \\
& \quad>\left(\rho \beta-l_{2}\right) \beta_{3}-\beta-M>0
\end{aligned}
$$

if $\rho>\frac{\beta+M}{\beta \beta_{3}}+\frac{l_{2}}{\beta}$. Here $M$ is defined in (3.6) and $l_{2}$ is as in (2.6). For $v<\delta_{1}$ or $v>1-\delta_{1}$, we have

$$
\left(\rho \beta+\int_{0}^{1} g^{\prime \prime}\left(v \pm \eta \delta e^{-\beta t}\right) \mathrm{d} \eta\right) v_{z}-\beta-\int_{0}^{1} f^{\prime}\left(v \pm \eta \delta e^{-\beta t}\right) \mathrm{d} \eta \geq \omega-\beta>0,
$$

if we set $0<\beta<\omega$ and $\rho>\frac{l_{2}}{\beta}$.

Take $\beta>0$ and $\rho>0$ such that $0<\beta<\omega$ and $\rho>\frac{\beta+M}{\beta \beta_{3}}+\frac{l_{2}}{\beta}$. Then we obtain $w_{t}^{+}+\mathcal{L}\left[w^{+}\right] \geq$ 0 and $w_{t}^{-}+\mathcal{L}\left[w^{-}\right] \leq 0$. Thus, we have proved that $w^{+}$and $w^{-}$are a supersolution and a subsolution, respectively. This completes the proof.

To prove the asymptotical stability of the traveling curved front $v_{*}$, we also need the following important auxiliary lemmas.

Lemma 3.4 Let $w(x, z, t)$ be the solution of (3.1) with (1.13). Then

$$
\lim _{R \rightarrow \infty} \sup _{x^{2}+z^{2} \geq R^{2}}\left|w(x, z, T)-v^{-}(x, z)\right|=0
$$

holds true for any fixed $T>0$.

Proof Define

$$
V(x, z)=U\left(\frac{z+\varphi(x)}{\sqrt{1+\varphi^{\prime 2}(x)}}\right) .
$$


By (1.8) and (2.1)-(2.4), we have

$$
\lim _{R \rightarrow \infty} \sup _{x^{2}+x^{2} \geq R^{2}}\left|v^{-}(x, z)-V(x, z)\right|=0,
$$

which combined with (1.13) implies

$$
\lim _{R \rightarrow \infty} \sup _{x^{2}+z^{2} \geq R^{2}}\left|u_{0}(x, z)-V(x, z)\right|=0 .
$$

Define

$$
W(x, z, t)=w(x, z, t)-V(x, z) .
$$

Then we have

$$
W_{t}-W_{x x}-W_{z z}+\left(s+g^{\prime}(W+V)\right) W_{z}-f(W+V)+f(V)=h(x, z, t)
$$

Here

$$
\begin{aligned}
h(x, z, t) & =-\mathcal{L}[V]-\left(g^{\prime}(W+V)-g^{\prime}(V)\right) V_{z} \\
& =V_{x x}+V_{z z}-\left(s+g^{\prime}(W+V)\right) V_{z}+f(V)
\end{aligned}
$$

satisfies

$$
\lim _{R \rightarrow \infty} \sup _{x^{2}+z^{2} \geq R^{2}}|h(x, z, t)|=0 \quad \text { uniformly for } t \geq 0 .
$$

Using $-f(W+V)+f(V)=-f^{\prime}(V+\ell W) W$ for some $0<\ell(x, z, t)<1$, we arrive at

$$
\left\{\begin{array}{l}
W_{t}-W_{x x}-W_{z z}+\left(s+g^{\prime}(W+V)\right) W_{z}-f^{\prime}(V+\ell W) W=h(x, z) \\
W(x, z, 0)=u_{0}(x, z)-V(x, z)
\end{array}\right.
$$

where $(x, z) \in \mathbb{R}^{2}$ and $t>0$. Let

$$
g_{1}(x, z, t)=-\left(s+g^{\prime}(W+V)\right), \quad g_{2}(x, z, t)=f^{\prime}(V+\ell W) .
$$

Instead of (3.13), we consider

$$
\left\{\begin{array}{l}
\tilde{W}_{t}=\tilde{W}_{x x}+\tilde{W}_{z z}+g_{1}(x, z, t) \tilde{W}_{z}+g_{2}(x, z, t) \tilde{W}+|h(x, z, t)|, \quad(x, z) \in \mathbb{R}^{2}, t>0 \\
\bar{W}(x, z, 0)=\left|u_{0}(x, z)-V(x, z)\right|, \quad(x, z) \in \mathbb{R}^{2}
\end{array}\right.
$$

Since $u_{0} \in B U C^{1}\left(\mathbb{R}^{2}\right)$, by the previous discussion we have that $g_{1}(x, z, t), g_{2}(x, z, t)$, and $h(x, z, t)$ are uniformly continuous in $(x, z, t) \in \mathbb{R}^{2} \times[0, \infty)$ and Hölder continuous in $(x, z) \in \mathbb{R}^{2}$ (the exponent is uniform for $(x, z, t) \in \mathbb{R}^{2} \times[0, \infty)$ ). Using the comparison principle, we easily get

$$
\tilde{W}(x, z, t) \geq|W(x, z, t)|, \quad \forall(x, z, t) \in \mathbb{R}^{2} \times[0, \infty)
$$


Friedman [18, Chapter 9, Theorem 2] implies that the fundamental solution $\Gamma\left(x, z, \xi_{1}, \xi_{2}\right.$, $t, \tau)$ of problem (3.14) satisfies

$$
\Gamma\left(x, z, \xi_{1}, \xi_{2}, t, \tau\right) \leq \frac{c_{1}}{t-\tau} e^{-c_{2} \frac{\left(x-\xi_{1}\right)^{2}+\left(z-\xi_{2}\right)^{2}}{t-\tau}} \quad \text { for } 0 \leq \tau<t \leq T
$$

where $c_{1}, c_{2}$ are positive constants depending only on $T$. Then the solution $\tilde{W}(x, z, t)$ of problem (3.14) can be decomposed as

$$
\tilde{W}(x, z, t)=I(x, z, t)+J(x, z, t)
$$

where

$$
\begin{aligned}
& I(x, z, t):=\int_{\mathbb{R}^{2}} \Gamma\left(x, z, \xi_{1}, \xi_{2}, t, 0\right) \tilde{W}(x, z, 0) \mathrm{d} \xi_{1} \mathrm{~d} \xi_{2} \\
& J(x, z, t):=\int_{0}^{t} \mathrm{~d} \tau \int_{\mathbb{R}^{2}} \Gamma\left(x, z, \xi_{1}, \xi_{2}, t, \tau\right)\left|h\left(\xi_{1}, \xi_{2}, \tau\right)\right| \mathrm{d} \xi_{1} \mathrm{~d} \xi_{2} .
\end{aligned}
$$

Then we have

$$
I \leq c_{1} \int_{\mathbb{R}^{2}} e^{-c_{2}\left(\eta_{1}^{2}+\eta_{2}^{2}\right)} \tilde{W}\left(x+\sqrt{t} \eta_{1}, z+\sqrt{t} \eta_{2}, 0\right) \mathrm{d} \eta_{1} \mathrm{~d} \eta_{2}
$$

On the other hand, there exists $0<t_{1}<t<T$ with

$$
J=t \int_{\mathbb{R}^{2}} \Gamma\left(x, z, \xi_{1}, \xi_{2}, t, t_{1}\right)\left|h\left(\xi_{1}, \xi_{2}, t_{1}\right)\right| \mathrm{d} \xi_{1} \mathrm{~d} \xi_{2}
$$

which yields

$$
J \leq c_{1} T \int_{\mathbb{R}^{2}} e^{-c_{2}\left(\eta_{1}^{2}+\eta_{2}^{2}\right)}\left|h\left(x+\sqrt{t-t_{1}} \eta_{1}, z+\sqrt{t-t_{1}} \eta_{2}, t_{1}\right)\right| \mathrm{d} \eta_{1} \mathrm{~d} \eta_{2} .
$$

Combining (3.15) and (3.16), we have $\lim _{R \rightarrow \infty} \sup _{x^{2}+z^{2} \geq R^{2}} \tilde{W}(x, z, T)=0$, which implies

$$
\lim _{R \rightarrow \infty} \sup _{x^{2}+z^{2} \geq R^{2}}|W(x, z, T)|=0
$$

for fixed $T>0$. Hence, we obtain

$$
\lim _{R \rightarrow \infty} \sup _{x^{2}+z^{2} \geq R^{2}}\left|w(x, z, T)-v^{-}(x, z)\right|=0 .
$$

This completes the proof.

Fix $\varepsilon \in\left(0, \frac{1}{2} \varepsilon_{0}^{+}\right)$and $\alpha \in\left(0, \alpha^{+}(\varepsilon)\right)$. By (2.27) and the comparison principle, we have

$$
v^{-}(x, z)<v_{*}(x, z)<w\left(x, z, t ; v^{+}\right)<v^{+}(x, z ; \varepsilon, \alpha) \quad \text { for }(x, z) \in \mathbb{R}^{2} \text { and } t>0 .
$$

Since $v^{+}(x, z ; \varepsilon, \alpha)$ is a supersolution of (1.9), we have that $w\left(x, z, t ; v^{+}\right)$is monotone decreasing in $t$ and the limit function

$$
v^{*}(x, z):=\lim _{t \rightarrow \infty} w\left(x, z, t ; v^{+}\right)
$$


exists. By the argument similar to that for $v_{*}$, we have that $v^{*}$ satisfies $\mathcal{L}\left[v^{*}\right]=0$ and

$$
\left\{\begin{array}{l}
v^{*}(-x, z)=v^{*}(x, z) \quad \text { for }(x, z) \in \mathbb{R}^{2}, \\
\frac{\partial}{\partial z} v^{*}(x, z)>0 \quad \text { for }(x, z) \in \mathbb{R}^{2}, \\
\frac{\partial}{\partial x} v^{*}(x, z)>0 \quad \text { for }(x, z) \in(0, \infty) \times \mathbb{R}, \\
v^{-}(x, z) \leq v_{*}(x, z) \leq v^{*}(x, z) \leq \min \left\{1, v^{+}(x, z ; \varepsilon, \alpha)\right\} \quad \text { for }(x, z) \in \mathbb{R}^{2} .
\end{array}\right.
$$

Lemma 3.5 Let $v_{*}$ and $v^{*}$ be as in (2.26) and (3.17). Then

$$
v_{*}(\cdot, \cdot) \equiv v^{*}(\cdot, \cdot) \quad \text { in } \mathbb{R}^{2} .
$$

The proof of the lemma is similar to that of Ninomiya and Taniguchi [43, Lemma 4.6], so we omit it. The following theorem shows that the traveling curved front $v_{*}$ is asymptotically stable for the initial data $u_{0} \in B U C^{1}\left(\mathbb{R}^{2}\right)$ with $u_{0} \geq v^{-}$.

Theorem 3.6 Let $u_{0}(x, z) \in B U C^{1}\left(\mathbb{R}^{2}\right)$ satisfy $v^{-}(x, z) \leq u_{0}(x, z)$ for $(x, z) \in \mathbb{R}^{2}$ and

$$
\lim _{R \rightarrow \infty} \sup _{x^{2}+z^{2} \geq R^{2}}\left|u_{0}(x, z)-v_{*}(x, z)\right|=0
$$

Then the solution $w\left(x, z, t ; u_{0}\right)$ of (3.1) satisfies

$$
\lim _{t \rightarrow \infty}\left\|w\left(\cdot, \cdot, t ; u_{0}\right)-v_{*}(\cdot, \cdot)\right\|_{L^{\infty}\left(\mathbb{R}^{2}\right)}=0 .
$$

Proof Denote $w\left(x, z, t ; u_{0}\right)$ by $w(x, z, t)$ for convenience. To complete the proof, it is sufficient to show that, for any $\varepsilon_{*}>0$, there exists a positive constant $T_{*}$ such that

$$
\sup _{(x, z) \in \mathbb{R}^{2}}\left|w(x, z, t)-v_{*}(x, z)\right| \leq \varepsilon_{*}, \quad \forall t>T_{*} .
$$

First, we choose $\delta$ small enough such that

$$
v^{*}(x, z+\rho \delta) \leq v^{*}(x, z)+\frac{\varepsilon_{*}}{3}, \quad 0<\delta<\varepsilon_{0}^{+},
$$

where $\varepsilon_{0}^{+}$and $\rho$ are defined in Lemma 2.2 and 3.3, respectively.

Next, we find a suitable supersolution. It follows from (3.3) that there exists $T_{\delta}>0$ with

$$
w\left(x, z, t ; v_{*}\right) \leq w(x, z, t)<1+\frac{\delta}{2}, \quad(x, z) \in \mathbb{R}^{2}, t \geq T_{\delta} .
$$

Lemma 3.4 implies that

$$
w\left(x, z, T_{\delta}\right) \leq v^{-}(x, z)+\frac{\delta}{2}, x^{2}+z^{2} \geq R^{2}
$$

for some $R>0$. Choose $\alpha$ small enough so that

$$
0<\alpha<\min \left\{\alpha_{0}^{+}(\delta), \frac{\varphi(0)}{R+\frac{s}{c} U^{-1}\left(1-\frac{\delta}{2}\right)}\right\} .
$$


Then we have

$$
\zeta=\frac{z+\varphi(\xi) / \alpha}{\sqrt{1+\varphi^{\prime}(\xi)^{2}}} \geq \frac{c}{s}(-R+\varphi(0) / \alpha) \geq U^{-1}\left(1-\frac{\delta}{2}\right)
$$

for $x^{2}+z^{2} \leq R^{2}$, and hence

$$
v^{+}(x, z) \geq 1-\frac{\delta}{2}, \quad x^{2}+z^{2} \leq R^{2},
$$

where $v^{+}(x, z)=v^{+}(x, z ; \delta, \alpha)$. From the above inequalities, we obtain

$$
w\left(x, z, T_{\delta}\right)<v^{+}(x, z)+\delta, \quad \forall(x, z) \in \mathbb{R}^{2} .
$$

It follows from Lemma 3.3 and the comparison principle that

$$
w\left(x, z, t+T_{\delta} ; v_{*}\right) \leq w\left(x, z, t+T_{\delta}\right) \leq w^{+}\left(x, z, t ; v^{+}\right), \quad t \geq 0 .
$$

Again applying Lemma 3.3 and the comparison principle, we obtain

$$
w\left(x, z, t+t^{\prime}+T_{\delta} ; v_{*}\right) \leq w\left(x, z, t+t^{\prime}+T_{\delta}\right) \leq w\left(x, z, t^{\prime} ; u^{t}\right)
$$

for $t^{\prime} \geq 0$, where

$$
u^{t}(x, z):=w^{+}\left(x, z, t ; v^{+}\right)
$$

Since $w\left(x, z, t ; v^{+}\right)$monotonically converges to $v^{*}(x, z)$ as $t \rightarrow \infty$, there exists a positive constant $t^{\prime \prime}$ with

$$
\sup _{(x, z) \in \mathbb{R}^{2}}\left|w\left(x, z, t^{\prime \prime} ; v^{+, \delta}\right)-v^{*}(x, z+\rho \delta)\right| \leq \frac{\varepsilon_{*}}{3},
$$

where

$$
v^{+, \delta}(x, z):=v^{+}(x, z+\rho \delta)
$$

Lemma 3.1 implies

$$
\left|w\left(x, z, t^{\prime \prime} ; u^{t}\right)-w\left(x, z, t^{\prime \prime} ; v^{+, \delta}\right)\right|_{C^{1}} \leq A_{0}^{t^{\prime \prime}+1}\left|u^{t}(x, z)-v^{+, \delta}(x, z)\right|_{C^{1}}
$$

where $A_{0}>1$ depends on $\left\|v^{+}\right\|_{C^{1}}$. Since $w^{+}\left(x, z, t ; v^{+}\right)=v^{+}\left(x, z+\rho \delta\left(1-e^{-\beta t}\right)\right)+\delta e^{-\beta t}$, $w_{x}^{+}\left(x, z, t ; v^{+}\right)=v_{x}^{+}\left(x, z+\rho \delta\left(1-e^{-\beta t}\right)\right)$, and $w_{z}^{+}\left(x, z, t ; v^{+}\right)=v_{z}^{+}\left(x, z+\rho \delta\left(1-e^{-\beta t}\right)\right)$, we can take $T_{1}>0$ large enough to satisfy

$$
A_{0}^{t^{\prime \prime}+1}\left|u^{t}(x, z)-v^{+, \delta}(x, z)\right|_{C^{1}}=A_{0}^{t^{\prime \prime}+1}\left|w^{+}\left(x, z, t ; v^{+}\right)-v^{+}(x, z+\rho \delta)\right|_{C^{1}} \leq \frac{\varepsilon_{*}}{3}
$$

for $t \geq T_{1}$. Combining (3.21) and (3.22), we have

$$
\left|w\left(x, z, t^{\prime \prime} ; u^{t}\right)-w\left(x, z, t^{\prime \prime} ; v^{+, \delta}\right)\right| \leq \frac{\varepsilon_{*}}{3}
$$


for $t \geq T_{1}$. Then, by (3.20) and (3.23), we get

$$
\begin{aligned}
& \left|w\left(x, z, t^{\prime \prime} ; u^{t}\right)-v^{*}(x, z+\rho \delta)\right| \\
& \quad \leq\left|w\left(x, z, t^{\prime \prime} ; u^{t}\right)-w\left(x, z, t^{\prime \prime} ; v^{+, \delta}\right)\right|+\left|w\left(x, z, t^{\prime \prime} ; v^{+, \delta}\right)-v^{*}(x, z+\rho \delta)\right| \leq \frac{2}{3} \varepsilon_{*}
\end{aligned}
$$

for any $t \geq T_{1}$, which implies

$$
w\left(x, z, t+t^{\prime \prime}+T_{\delta}\right) \leq w\left(x, z, t^{\prime \prime} ; u^{t}\right) \leq v^{*}(x, z+\rho \delta)+\frac{2}{3} \varepsilon_{*}, \quad \forall t \geq T_{1} .
$$

By (3.18), (3.19), (3.24), and Lemma 3.5, we obtain

$$
w\left(x, z, t ; v_{*}\right) \leq w(x, z, t) \leq v^{*}(x, z)+\varepsilon_{*}=v_{*}(x, z)+\varepsilon_{*}
$$

for $(x, z) \in \mathbb{R}^{2}$ and $t \geq t^{\prime \prime}+T_{1}+T_{\delta}$. Let $T_{*}:=t^{\prime \prime}+T_{1}+T_{\delta}$. Since $v_{*}(x, z)=\lim _{t \rightarrow \infty} w\left(x, z, t ; v_{*}\right)$, we have $v_{*}(x, z) \leq w(x, z, t) \leq v_{*}(x, z)+\varepsilon_{*}$ for all $(x, z) \in \mathbb{R}^{2}$ and $t>T_{*}$. This completes the proof.

Remark 3.7 Combining Theorems 2.3 and 3.6, we can complete the proof of Theorem 1.1. Theorem 3.6 also asserts that $v_{*}$ is a unique traveling curved front satisfying (1.10) and (1.12).

\section{Discussion}

In this paper, under assumptions (F) and (G), we establish the existence and stability of traveling curved front $v_{*}$ of $(1.1)$ in $\mathbb{R}^{2}$ for every direction $\theta \in(0, \pi / 2)$ satisfying $(C)$. For such a reaction-convection-diffusion equation, as mentioned in the first section, the planar traveling wave profile $U_{\theta}$ of (1.1) and the corresponding wave speed $c_{\theta}$ depend on the propagation direction $\theta \in[0,2 \pi)$. Clearly, in this paper we only consider a simple convection term $(g(u))_{y}=\nabla \cdot(0, g(u))$, namely, it is supposed that the nonlinear convection only occurs in the $y$-direction. Let $U_{\theta}\left(x \cos \theta+y \sin \theta+c_{\theta} t\right)$ be the traveling wave front of (1.1) along the direction $\theta \in(0, \pi / 2)$ (or $(\cos \theta, \sin \theta)$ ). Due to such an assumption, we always have that $U_{\theta}\left(-x \cos \theta+y \sin \theta+c_{\theta} t\right)$ is a planar traveling wave front of (1.1) along the direction $\pi-\theta$ (or $(-\cos \theta, \sin \theta))$. Hence, we can prove the main results of this paper by using the method similar to those in Ninomiya and Taniguchi [43] and Wang [54]. Beyond all doubt, it is more reasonable to consider the following convection term:

$$
\nabla \cdot(h(u), g(u))
$$

But in this case, the function $U_{\theta}\left(-x \cos \theta+y \sin \theta+c_{\theta} t\right)$ is no longer a traveling wave front of the equation along the direction $\pi-\theta$ (or $(-\cos \theta, \sin \theta)$ ). Thus, the supersolution constructed in Lemma 2.2 does not work in this case and we cannot get the existence and stability of traveling curved fronts by the arguments of this paper. Therefore, to consider traveling curved fronts of $(1.1)$ with a convection term $\nabla \cdot(h(u), g(u))$ is a very interesting and difficult problem, and we leave it as a future work.

Here we also would like to give more comments on conditions (F)(iii) and (C). In fact, for every $\theta \in[0,2 \pi)$, the existence of traveling wave front $U_{\theta}\left(x \cos \theta+y \sin \theta+c_{\theta} t\right)$ of (1.1) 
follows from conditions (F)(i), (F)(ii), (F)(iv), and (G). Consequently, we can get $c_{0}>0$ by condition (F)(iii). As discussed in Sect. 1, it follows from $c_{0}>0$ that there exists a subset of $(0, \pi / 2)$ in which every $\theta$ satisfies condition $(C)$ (at least, there exists $\theta^{*} \in(0, \pi / 2)$ such that each $\theta \in\left[0, \theta^{*}\right)$ satisfies condition $\left.(C)\right)$. On this basis, for each $\theta \in(0, \pi / 2)$ which satisfies $(C)$, we can establish the corresponding traveling curved front $v_{*}\left(x, y+s_{\theta} t\right)$ with speed $s_{\theta}=\frac{c}{\sin \theta}$, see Theorem 1.1. Clearly, to establish the existence of traveling curved fronts by the method of this paper, the supersolution constructed in Lemma 2.2 plays a crucial role. Observing the proof of Lemma 2.2, we find that inequality (2.12) seems indispensable. Thus, condition (C) is necessary for using the method of this paper to establish the existence of traveling curved fronts. By a direct calculation, we have

$$
\int_{0}^{1} f(r) \mathrm{d} r=\int_{-\infty}^{+\infty}\left(c_{\theta}+g^{\prime}\left(U_{\theta}(r)\right) \sin \theta\right)\left(U_{\theta}^{\prime}(r)\right)^{2} \mathrm{~d} r .
$$

Under assumption (F)(iii), the inequality $c_{\theta}+\sup _{r \in[0,1]} g^{\prime}(r) \sin \theta<0$ cannot hold, because the inequality implies that $\int_{0}^{1} f(r) \mathrm{d} r<0$. Thus, under conditions (F) and (G), for $\theta \in(0, \pi / 2)$ which does not satisfy (C), do traveling curved fronts of (1.1) exist or not? How to establish the traveling curved front of (1.1) in this case? These are very interesting questions.

\section{Acknowledgements}

The authors would like to express thanks to anonymous reviewers for their excellent suggestions.

\section{Funding}

This work was supported by the National Natural Science Foundation of China (NO. 11701012), the NSF of Ningxia Hui Autonomous Region of China (NO. 2018AAC03129), and the General Research Projects of North Minzu University (NO. 2020XYZSX03) and the First-Class Disciplines Foundation of Ningxia (NO. NXYLXK2017B09).

Availability of data and materials

Not applicable.

\section{Competing interests}

The authors declare that they have no competing interests.

\section{Authors' contributions}

All authors contributed equally and significantly in writing this article. All authors read and approved the final manuscript.

\section{Publisher's Note}

Springer Nature remains neutral with regard to jurisdictional claims in published maps and institutional affiliations.

Received: 28 February 2020 Accepted: 18 August 2020 Published online: 10 September 2020

\footnotetext{
References

1. Berestycki, H.: The influence of advection on the propagation of fronts in reaction-diffusion equations. In: Berestycki, H., Pomeau, Y. (eds.) Nonlinear PDEs in Condensed Matter and Reactive Flows. NATO Sci. Ser. C, vol. 569. Kluwer, Dordrecht (2003)

2. Bonnet, A., Hamel, F.: Existence of nonplanar solutions of a simple model of premixed Bunsen flames. SIAM J. Math. Anal. 31, 80-118 (1999)

3. Brazhnik, P.K., Tyson, J.J.: On traveling wave solutions of Fisher's equation in two spatial dimensions. SIAM J. Appl. Math. 60, 371-391 (2000)

4. Bu, Z.-H., Ma, L.-Y., Wang, Z.-C.: Stability of pyramidal traveling fronts in the degenerate monostable and combustion equations II. Nonlinear Anal., Real World Appl. 47, 80-118 (2019)

5. Bu, Z.-H., Ma, L.-Y., Wang, Z.-C.: Conical traveling fronts of combustion equations in R33. Appl. Math. Lett. 108, Article ID $106509(2020)$

6. Cencini, M., Lopez, C., Vergni, D.: Reaction-diffusion systems: front propagation and spatial structures. In: The Kolmogorov Legacy in Physics. Lecture Notes in Phys., vol. 636, pp. 187-210. Springer, Berlin (2003)

7. Chen, X., Guo, J.-S., Hamel, F., Ninomiya, H., Roquejoffre, J.-M.: Traveling waves with paraboloid like interfaces for balanced bistable dynamics. Ann. Inst. Henri Poincaré, Anal. Non Linéaire 24, 369-393 (2007)

8. Crooks, E.C.M.: Stability of travelling-wave solutions for reaction-diffusion-convection systems. Topol. Methods Nonlinear Anal. 16, 37-63 (2000)
} 
9. Crooks, E.C.M.: Travelling fronts for monostable reaction-diffusion systems with gradient-dependence. Adv. Differ. Equ. 8, 279-314 (2003)

10. Crooks, E.C.M.: Front profiles in the vanishing-diffusion limit for monostable reaction-diffusion-convection equations. Differ. Integral Equ. 23, 495-512 (2010)

11. Crooks, E.C.M., Mascia, C.: Front speeds in the vanishing diffusion limit for reaction-diffusion-convection equations. Differ. Integral Equ. 20, 499-514 (2007)

12. Crooks, E.C.M., Toland, J.F.: Travelling waves for reaction-diffusion-convection systems. Topol. Methods Nonlinear Anal. 11, 19-43 (1998)

13. Crooks, E.C.M., Tsai, J.-C.: Front-like entire solutions for equations with convection. J. Differ. Equ. 253, 1206-1249 (2012)

14. Daners, D., Koch Medina, P.: Abstract Evolution Equations, Periodic Problems and Applications. Pitman Research Notes in Mathematics Series, vol. 279. Longman, Harlow; copublished in the United States with Wiley, New York (1992)

15. Del Pino, M., Kowalczyk, M., Wei, J.: Traveling waves with multiple and non-convex fronts for a bistable semilinear parabolic equation. Commun. Pure Appl. Math. 66, 481-547 (2013)

16. El Smaily, M., Hamel, F., Huang, R.: Two-dimensional curved fronts in a periodic shear flow. Nonlinear Anal., Theory Methods Appl. 74, 6469-6486 (2011)

17. Feng, B.: On the blow-up solutions for the nonlinear Schrödinger equation with combined power-type nonlinearities. J. Evol. Equ. 18, 203-220 (2018)

18. Friedman, A.: Partial Differential Equations of Parabolic Type. Prentice-Hall, Englewood Cliffs (1964)

19. Gilbarg, D., Trudinger, N.S.: Elliptic Partial Differential Equations of Second Order, 2nd edn. Grundlehren der Mathematischen Wissenschaften [Fundamental Principles of Mathematical Sciences], vol. 224. Springer, Berlin (1983)

20. Gilding, B.H.: On front speeds in the vanishing diffusion limit for reaction-convection-diffusion equations. Differ. Integral Equ. 23, 445-450 (2010)

21. Gilding, B.H., Kersner, R.: Travelling Waves in Nonlinear Diffusion-Convection Reaction. Progr. Nonlinear Differential Equations Appl., vol. 60. Birkhäuser, Basel (2004)

22. Gui, C.: Symmetry of traveling wave solutions to the Allen-Cahn equation in $\mathbb{R}^{2}$. Arch. Ration. Mech. Anal. 203, 1037-1065 (2012)

23. Hamel, F.: Bistable transition fronts in $\mathbb{R}^{N}$. Adv. Math. 289, 279-344 (2016)

24. Hamel, F., Monneau, R.: Solutions of semilinear elliptic equations in $\mathbb{R}^{N}$ with conical-shaped level sets. Commun. Partial Differ. Equ. 25, 769-819 (2000)

25. Hamel, F., Monneau, R., Roquejoffre, J.-M.: Stability of travelling waves in a model for conical flames in two space dimensions. Ann. Sci. Éc. Norm. Supér. 37, 469-506 (2004)

26. Hamel, F., Monneau, R., Roquejoffre, J.-M.: Existence and qualitative properties of multidimensional conical bistable fronts. Discrete Contin. Dyn. Syst. 13, 1069-1096 (2005)

27. Hamel, F., Monneau, R., Roquejoffre, J.-M.: Asymptotic properties and classification of bistable fronts with Lipschitz level sets. Discrete Contin. Dyn. Syst. 14, 75-92 (2006)

28. Hamel, F., Nadirashvili, N.: Travelling fronts and entire solutions of the Fisher-KPP equation in $\mathbb{R}^{N}$. Arch. Ration. Mech. Anal. 157, 91-163 (2001)

29. Hamel, F., Roquejoffre, J.M.: Heteroclinic connections for multidimensional bistable reaction-diffusion equations. Discrete Contin. Dyn. Syst., Ser. S 4, 101-123 (2011)

30. Haragus, M., Scheel, A.: Almost planar waves in anisotropic media. Commun. Partial Differ. Equ. 31, $791-815$ (2006)

31. Haragus, M., Scheel, A.: Corner defects in almost planar interface propagation. Ann. Inst. Henri Poincaré, Anal. Non Linéaire 23, 283-329 (2006)

32. Huang, R.: Stability of travelling fronts of the Fisher-KPP equation in $\mathbb{R}^{N}$. NoDEA Nonlinear Differ. Equ. Appl. 15 , 599-622 (2008)

33. Kurokawa, Y., Taniguchi, M.: Multi-dimensional pyramidal travelling fronts in the Allen-Cahn equations. Proc. R. Soc. Edinb., Sect. A 141, 1031-1054 (2011)

34. Ladyženskaja, O.A., Solonnikov, V.A., Ural'ceva, N.N.: Linear and Quasilinear Equations of Parabolic Type. Translations of Mathematical Monographs, vol. 23. Am. Math. Soc., Providence (1968) (Russian). Translated from the Russian by S. Smith

35. Lunardi, A.: Analytic Semigroups and Optimal Regularity in Parabolic Problems. Birkhäuser, Basel (1995)

36. Malaguti, L., Marcelli, C.: Travelling wavefronts in reaction-diffusion equations with convection effects and non-regular terms. Math. Nachr. 242, 148-164 (2002)

37. Malaguti, L., Marcelli, C.: The influence of convective effects on front propagation in certain diffusive models. In: Capasso, V. (ed.) Mathematical Modelling and Computing in Biology and Medicine: 5th ESMTB Conference 2002. Esculapio, Bologna, pp. 362-367 (2003)

38. Malaguti, L., Marcelli, C., Matucci, S.: Front propagation in bistable reaction-diffusion-advection equations. Adv. Differ. Equ. 9, 1143-1166 (2004)

39. Mascia, C.: Travelling wave solutions for a balance law. Proc. R. Soc. Edinb., Sect. A 127, 567-593 (1997)

40. Mascia, C.: Qualitative behavior of conservation laws with reaction term and nonconvex flux. Q. Appl. Math. 58 739-761 (2000)

41. Murray, J.D.: Mathematical Biology, vol. 19. Springer, Berlin (1989)

42. Ni, W.-M., Taniguchi, M.: Traveling fronts of pyramidal shapes in competition-diffusion systems. Netw. Heterog. Media 8, 379-395 (2013)

43. Ninomiya, H., Taniguchi, M.: Existence and global stability of traveling curved fronts in the Allen-Cahn equations. J. Differ. Equ. 213, 204-233 (2005)

44. Ninomiya, H., Taniguchi, M.: Global stability of traveling curved fronts in the Allen-Cahn equation. Discrete Contin. Dyn. Syst. 15, 819-832 (2006)

45. Niu, H.-T., Bu, Z.-H., Wang, Z.-C.: Global stability of curved fronts in the Belousov-Zhabotinskii reaction-diffusion system in $R^{2}$. Nonlinear Anal., Real World Appl. 46, 493-524 (2019)

46. Niu, H.-T., Wang, Z.-C., Bu, Z.-H.: Curved fronts in the Belousov-Zhabotinskii reaction-diffusion systems in $R^{2}$. J. Differ. Equ. 264, 5758-5801 (2018) 
47. Sheng, W.-J., Li, W.-T., Wang, Z.-C.: Multidimensional stability of V-shaped traveling fronts in the Allen-Cahn equation. Sci. China Math. 56, 1969-1982 (2013)

48. Smith, H.L., Zhao, X.-Q.: Global asymptotic stability of travelling waves in delayed reaction-diffusion equations. SIAM J. Math. Anal. 31, 514-534 (2000)

49. Taniguchi, M.: Traveling fronts of pyramidal shapes in the Allen-Cahn equations. SIAM J. Math. Anal. 39, 319-344 (2007)

50. Taniguchi, M.: The uniqueness and asymptotic stability of pyramidal traveling fronts in the Allen-Cahn equations. J. Differ. Equ. 246, 2103-2130 (2009)

51. Taniguchi, M.: Multi-dimensional traveling fronts in bistable reaction-diffusion equations. Discrete Contin. Dyn. Syst. 32, 1011-1046 (2012)

52. Volpert, A.I., Volpert, V.A., Volpert, V.A.: Traveling Wave Solutions of Parabolic Systems, vol. 140. Am. Math. Soc., Providence (1994)

53. Wang, J., Cao, J.-F.: Fujita type critical exponent for a free boundary problem with spatial-temporal source. Nonlinear Anal., Real World Appl. 51, Article ID 103004 (2020)

54. Wang, Z.-C.: Traveling curved fronts in monotone bistable systems. Discrete Contin. Dyn. Syst. 32, 2339-2374 (2012)

55. Wang, Z.-C.: Cylindrically symmetric traveling fronts in periodic reaction-diffusion equations with bistable nonlinearity. Proc. R. Soc. Edinb., Sect. A 145A, 1053-1090 (2015)

56. Wang, Z.-C., Bu, Z.-H.: Nonplanar traveling fronts in reaction-diffusion equations with combustion and degenerate Fisher-KPP nonlinearities. J. Differ. Equ. 260, 6405-6450 (2016)

57. Wang, Z.-C., Li, W.-T., Ruan, S.: Existence and stability of traveling wave fronts in reaction advection diffusion equations with nonlocal delay. J. Differ. Equ. 238, 153-200 (2007)

58. Wang, Z.-C., Li, W.-T., Ruan, S.: Existence, uniqueness and stability of pyramidal traveling fronts in reaction-diffusion systems. Sci. China Math. 59, 1869-1908 (2016)

59. Wang, Z.-C., Niu, H.-L., Ruan, S.: On the existence of axisymmetric traveling fronts in Lotka-Volterra competition-diffusion systems in $R^{3}$. Discrete Contin. Dyn. Syst., Ser. B 22, 1111-1144 (2017)

60. Wang, Z.-C., Wu, J.: Periodic traveling curved fronts in reaction-diffusion equation with bistable time-periodic nonlinearity. J. Differ. Equ. 250, 3196-3229 (2011)

\section{Submit your manuscript to a SpringerOpen ${ }^{\circ}$ journal and benefit from:}

- Convenient online submission

- Rigorous peer review

- Open access: articles freely available online

- High visibility within the field

- Retaining the copyright to your article

Submit your next manuscript at $>$ springeropen.com 